\title{
Redistribution of particles across the nucleus of comet 67P/Churyumov-Gerasimenko
}

\author{
N. Thomas ${ }^{1}$, B. Davidsson ${ }^{2}$, M. R. El-Maarry ${ }^{1}$, S. Fornasier ${ }^{3}$, L. Giacomini ${ }^{4}$, A. G. Gracia-Berná1 ${ }^{1}$, S. F. Hviid ${ }^{5}$, \\ W.-H. Ip ${ }^{6}$, L. Jorda ${ }^{7}$, H. U. Keller ${ }^{8,5}$, J. Knollenberg ${ }^{5}$, E. Kührt ${ }^{5}$, F. La Forgia ${ }^{12}$, I. L. Lai ${ }^{6}$, Y. Liao ${ }^{1}$, R. Marschall ${ }^{1}$, \\ M. Massironi ${ }^{4}$, S. Mottola ${ }^{5}$ M. Pajola ${ }^{9}$, O. Poch ${ }^{1}$, A. Pommerol ${ }^{1}$, F. Preusker ${ }^{5}$, F. Scholten ${ }^{5}$, C. C. Su ${ }^{10}$, J. S. Wu ${ }^{10}$, \\ J.-B. Vincent ${ }^{11}$, H. Sierks ${ }^{11}$, C. Barbieri ${ }^{12}$, P. L. Lamy ${ }^{7}$, R. Rodrigo ${ }^{13}$, D. Koschny ${ }^{14}$, H. Rickman ${ }^{15}$, M. F. A'Hearn ${ }^{16}$, \\ M. A. Barucci ${ }^{3}$, J-L. Bertaux ${ }^{17}$, I. Bertini ${ }^{12}$, G. Cremonese ${ }^{18}$, V. Da Deppo ${ }^{19}$, S. Debei ${ }^{20}$, M. de Cecco ${ }^{21}$, M. Fulle 22 , \\ O. Groussin ${ }^{7}$, P. J. Gutierrez ${ }^{23}$, J.-R. Kramm ${ }^{11}$, M. Küppers ${ }^{24}$, L. M. Lara ${ }^{23}$, M. Lazzarin ${ }^{12}$, J. J. Lopez Moreno ${ }^{23}$, \\ F. Marzari ${ }^{12}$, H. Michalik ${ }^{25}$, G. Naletto ${ }^{9,18,19}$, J. Agarwal ${ }^{11}$, C. Güttler ${ }^{11}$, N. Oklay ${ }^{11}$, and C. Tubiana ${ }^{11}$
}

(Affiliations can be found after the references)

Received 7 March 2015 / Accepted 9 June 2015

\begin{abstract}
Context. We present an investigation of the surface properties of areas on the nucleus of comet 67P/Churyumov-Gerasimenko.

Aims. We aim to show that transport of material from one part of the cometary nucleus to another is a significant mechanism that influences the appearance of the nucleus and the surface thermal properties.

Methods. We used data from the OSIRIS imaging system onboard the Rosetta spacecraft to identify surface features on the nucleus that can be produced by various transport mechanisms. We used simple calculations based on previous works to establish the plausibility of dust transport from one part of the nucleus to another.

Results. We show by observation and modeling that "airfall" as a consequence of non-escaping large particles emitted from the neck region of the nucleus is a plausible explanation for the smooth thin deposits in the northern hemisphere of the nucleus. The consequences are also discussed. We also present observations of aeolian ripples and ventifacts. We show by numerical modeling that a type of saltation is plausible even under the rarified gas densities seen at the surface of the nucleus. However, interparticle cohesive forces present difficulties for this model, and an alternative mechanism for the initiation of reptation and creep may result from the airfall mechanism. The requirements on gas density and other parameters of this alternative make it a more attractive explanation for the observations. The uncertainties and implications are discussed.
\end{abstract}

Key words. space vehicles: instruments - comets: individual: 67P/Churyumov-Gerasimenko - techniques: image processing - hydrodynamics

\section{Introduction}

The European Space Agency's Rosetta spacecraft entered orbit around the nucleus of the Jupiter-family comet, 67P/Churyumov-Gerasimenko (hereafter 67P) on 6 August 2014. The scientific imaging system onboard is called OSIRIS (Keller et al. 2007) and comprises a dual camera system with a high-resolution (scale $=18.56 \mu \mathrm{rad} / \mathrm{px}$ ) narrow-angle camera (NAC) and a lower resolution $(101 \mu \mathrm{rad} / \mathrm{px})$ wide-angle camera (WAC). Initial results from OSIRIS observations of the nucleus and the innermost coma have been published in Sierks et al. (2015) and Thomas et al. (2015).

Sublimation-driven ejection of material from an active area is the initiator of material motion in a cometary system. The nonvolatile material (usually referred to as dust) is accelerated by gas, and much of it reaches escape velocity, thereby permanently leaving the cometary environment. However, it is now clear that not all non-volatile material acquires sufficient energy to escape. As discussed in the following section, there is evidence in the images for the accumulation of non-escaping dust particles emitted from areas of activity in a form of "airfall"1. Furthermore, there

\footnotetext{
1 We use the term "airfall" by analogy with volcanic products to mean the deposition of material ejected from a vent or similar.
}

are observations of features that appear similar to aeolian ripples, dune-like structures, and wind-tails, indicating that other processes may be at work in transporting non-volatile material across the surface. In addition, there are smooth depressions that appear similar to what has been inferred to be ponded dust deposits on asteroid 433 Eros (Robinson et al. 2001). These observations suggest that surface dust transport ${ }^{2}$ is of major importance in defining the uppermost surface layer in many regions (Thomas et al. 2015).

In this paper, we present evidence for motion of material from one site on the nucleus to another. In the following section, we examine the evidence for airfall. In Sect. 3, we present a simple model using the rather complex gravitational potential and discuss the somewhat unusual effects resulting from emission at the neck for the observed airfall deposits. We also use a gas dynamics model to estimate particle escape probabilities at the considered heliocentric distances. In Sect. 4, we study the possible consequences of airfall, and in Sect. 5, we present evidence for surface ventifacts (including the remarkable observation of

2 It is important to recognize that the particle sizes involved in this transport are likely to be in the millimeter- to decimeter-size range and hence should be referred to as coarse sand (following Wentworth) or fines instead of the generic term of cometary dust. 
what appear to be aeolian ripples). In Sect. 6, we discuss the feasibility of wind-driven transport on the nucleus in the presence of cohesive forces using a gas dynamics model. In Sect. 7, describe the ponded deposits on the nucleus, which are prevalent in several regions. In Sect. 8, we discuss some of the consequences of the observations and conclude.

Throughout, we use the regional nomenclature previously outlined in Thomas et al. (2015) and recently expanded upon by El-Maarry et al. (2015).

\section{Evidence of airfall}

\subsection{Introduction}

The idea of particles emitted from active regions failing to escape the gravitational field of a cometary nucleus has been explored on several occasions. For example, Richter \& Keller (1995) produced a semi-analytical model that was used to determine the number densities of larger particles on bound orbits in the vicinity of the nucleus. It was shown that only particles of about $5 \mathrm{~cm}$ in size could achieve stable orbits. The ultimate aim here was to establish the probabilities of bound particles impacting an orbiting spacecraft. Similar calculations were performed by Fulle (1997). Bound particles may either escape or re-impact the surface if further perturbations are applied.

A little earlier, Moehlmann (1994) had argued that cm- and dm-sized particles could fall back if they do not acquire sufficient energy, thereby producing a loosely packed "deposition regolith". Kührt et al. (1997) identified airfall (referred to there as "dust hail") as a potential risk to cometary landers and showed that the $\mathrm{cm}$-sized particles would be the main contributors to the surface coverage (as envisaged by Moehlmann 1994), although the assumptions made were somewhat uncertain. These works had identified that gas drag on larger particles may not be sufficient to accelerate them beyond escape velocity, but they also pointed out that local variations in activity (i.e., the presence of active and non-active regions in close proximity) would naturally lead to additional airfall as particles left highdensity regions in the flow field, resulting in negligible further acceleration.

In general, these discussions considered steady-state gas emission, but from irregularly distributed active sources. On the other hand, quasi-explosive events may also be of importance. It has been postulated that dust emission can be driven, particularly at high heliocentric distances, by localized sublimation of supervolatiles such as $\mathrm{CO}$, and $\mathrm{CO}_{2}$, or the amorphous-crystalline ice transition (Prialnik \& Bar-Nun 1990). The build-up of pressure in the sub-surface by super-volatile sublimation can lead to ejection of larger particles through quasi-explosive events. This might lead to emission, but also to extremely rapid decoupling from the gas flow, which would result in low velocities of the ejecta relative to the nucleus.

Conceptually, airfall might therefore be expected as a result of several similar, but slightly different processes. We show in the following sections key observations supporting the importance of airfall. They are (1) observations of surface deposits; (2) slow-moving particles in bound orbits; (3) slow-moving particles at the bases of jet-like features; and (4) observations of bright icy chunks on the surface.

\subsection{Surface deposits}

There are four regions on the nucleus of $67 \mathrm{P}$ that show evidence of a surface deposit: Ash and Babi on the "body" of the nucleus,
Ma'at on the "head", and Seth, which is on the body, but immediately adjacent to the "neck". Some other regions also show small patches of similar deposits, for instance, in Anuket close to the border with Ma'at. The global distribution of these smooth deposits on the nucleus can be seen in Fig. 1. The smooth deposits in other areas (notably Anubis and Imhotep; Auger et al. 2015) have a completely different appearance with possible layering that has not been observed elsewhere.

In these regions, surfaces that are roughly facing north are relatively smooth, but adjacent vertical surfaces are rough and fractured. In Fig. 2, we show an example from the Ash region. The pit-like depression has steep walls. On the pit floor and on the surrounding terrain, the surface is smooth at the resolution of the presented image. (At higher resolution, the surface has a rougher more inhomogeneous appearance, as we discuss in the next subsection.) There is no deposit on the walls of the pit. The wall is fractured with vertical lineaments. Figure 2 gives the strong impression that the smooth material is a rather thin veneer over the fractured material. The thickness of the thin material at the edge of the pit seems to be close to the resolution limit $(0.34 \mathrm{~m} / \mathrm{px})$. The rougher terrain seen in the upper right corner of the image is covered to some extent by smooth material, but has not been buried by it. There is some evidence of collapse of the pit wall with talus at the base.

Thomas et al. (2015) showed a cut of the flat-floored pit at the interface of Seth and Hapi, with an apparently dusty coating on a horizontal surface with the adjacent nearly vertical surface being visually clean of this coating (their Fig. 2; right). Here again a deposition process from above is an attractive explanation. In Fig. 3 we show the same feature, but from a direction almost orthogonal to the vertical face. This again illustrates that the smooth layer must be rather thin.

The layer is not, however, the most recent feature on the nucleus. In Fig. 4 we show an image of the Ash region where the smooth layer is draped over the material below. Here, however, the quasi-vertical part of the surface has been disrupted and talus has accumulated at the base. Boulders produced by this mass wasting are located on the smooth layer below. This process appears to be continuing. Zooming-in to the edge (Fig. 5), cleaving of the upper surface is visible, which will probably result in additional collapse. Positions in the image that show the fractured material below the smooth upper layer again indicate a thin layer of smooth material.

Thomas et al. (2015) identified a possible impact crater (their Fig. S2) that appears partially buried by the smooth material. Estimates of the original crater depth or diameter ratio lead to smooth material thicknesses of 1-5 m, which suggests that although the layer is thin, it may be thinnest at the edges, but with greater thickness elsewhere. However, there is no evidence that the deposit is thicker than $5 \mathrm{~m}$.

We can use this information to try to estimate a total volume of the smooth material. The bulk area of Ash, Babi, Ma'at, and Seth together is $\approx 8 \mathrm{~km}^{2}$. Assuming a layer of one meter thickness, we obtain a volume of $0.008 \mathrm{~km}^{3}$. If we furthermore assume that the material has a bulk density of $1000 \mathrm{~kg} / \mathrm{m}^{3}$, then the layer has a mass of $8 \times 10^{9} \mathrm{~kg}$, which would be roughly equivalent to the total mass lost by the comet in two orbits about the Sun. For comparison, the neck region of the nucleus is around $2.2 \mathrm{~km}$ long, roughly $800 \mathrm{~m}$ wide, and might be considered to be $1 \mathrm{~km}$ deep. This crude calculation shows that if activity at the neck were the only source for the smooth material and if the comet were originally a more regular ellipsoidal form, then less than $0.5 \%$ of the material emitted from what we now see as the neck would need to find its way into the deposits to produce what 


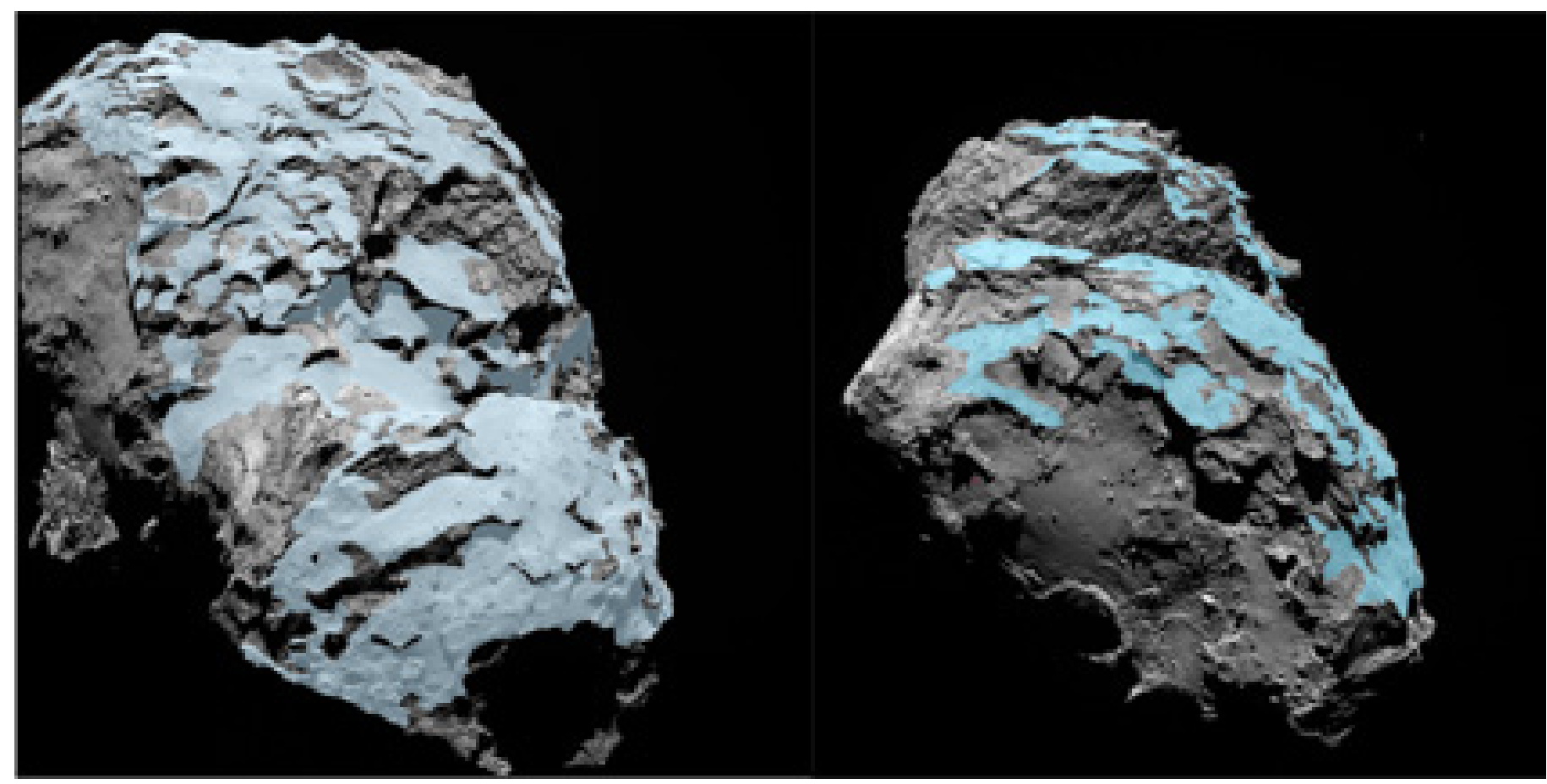

Fig. 1. Positions and areas of smooth deposits on the nucleus seen in two orientations.

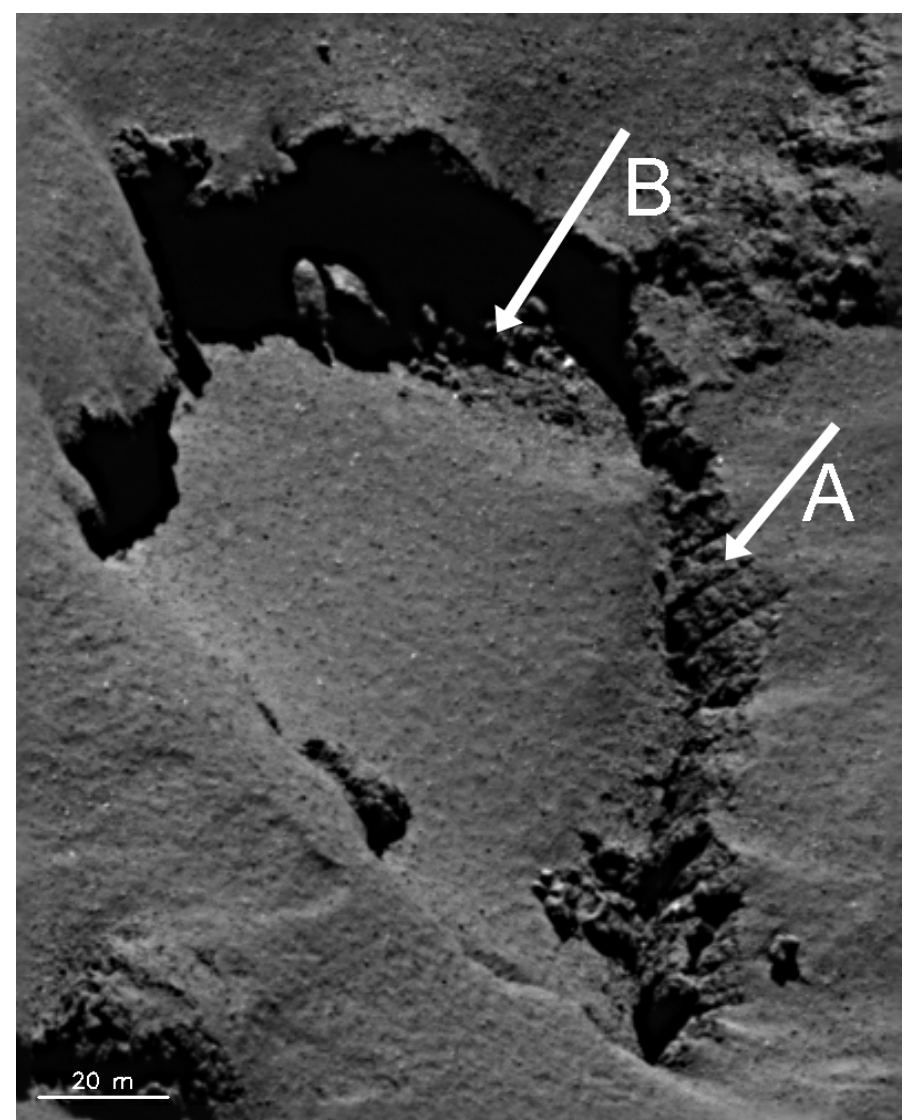

Fig. 2. Pit in the Ash region. The pit floor and adjacent terrain are smooth. The pit walls are fractured and relatively clean (position A); talus was presumably produced by wall collapse (position B). Image NAC_2014-10-01T02.43.53.558Z_ID10_1397549300_F22

1 we currently see. This appears to be plausible and might suggest 2 that no additional source is needed. However, we note that the

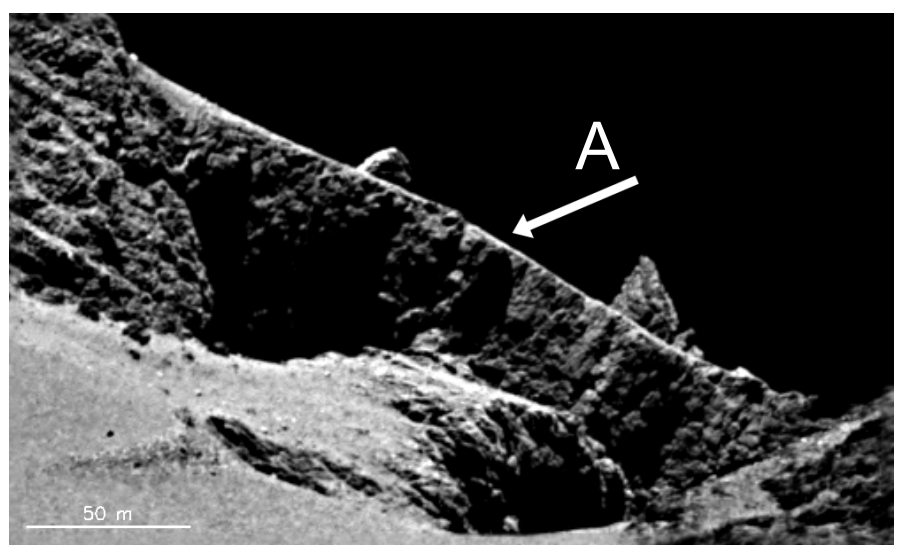

Fig. 3. View of the cut with a diameter of $600 \mathrm{~m}$ of the flat-floored pit at the interface of Seth and Hapi. This view is almost orthogonal to the vertical face and shows that the dust and fines covering are extremely thin (position A) at the resolution of the NAC. Image: NAC_2014-0917T23.52.43.330Z_ID10_1397549400_F22.

southern hemisphere will become more active near perihelion as a result of the increased insolation and the obliquity (Keller et al. 2015), so that deposition from this source is conceivable.

\subsection{Evidence of slow-moving particles}

\subsubsection{In bound orbits}

Rotundi et al. (2015) has provided evidence of slow-moving particles in bound orbits about the nucleus of 67P. Given the surface gravitational acceleration of roughly $\approx 1.6 \times 10^{-4} \mathrm{~m} / \mathrm{s}^{2}$, this implies ejection velocities of $<0.8 \mathrm{~m} / \mathrm{s}$ for significant amounts of material. This has followed observations by the Deep Impact spacecraft (as part of the EPOXI mission) that comet $103 \mathrm{P} /$ Hartley 2 was surrounded by debris composed of fine grained dust, ice, and hundreds of discrete millimeter- to 


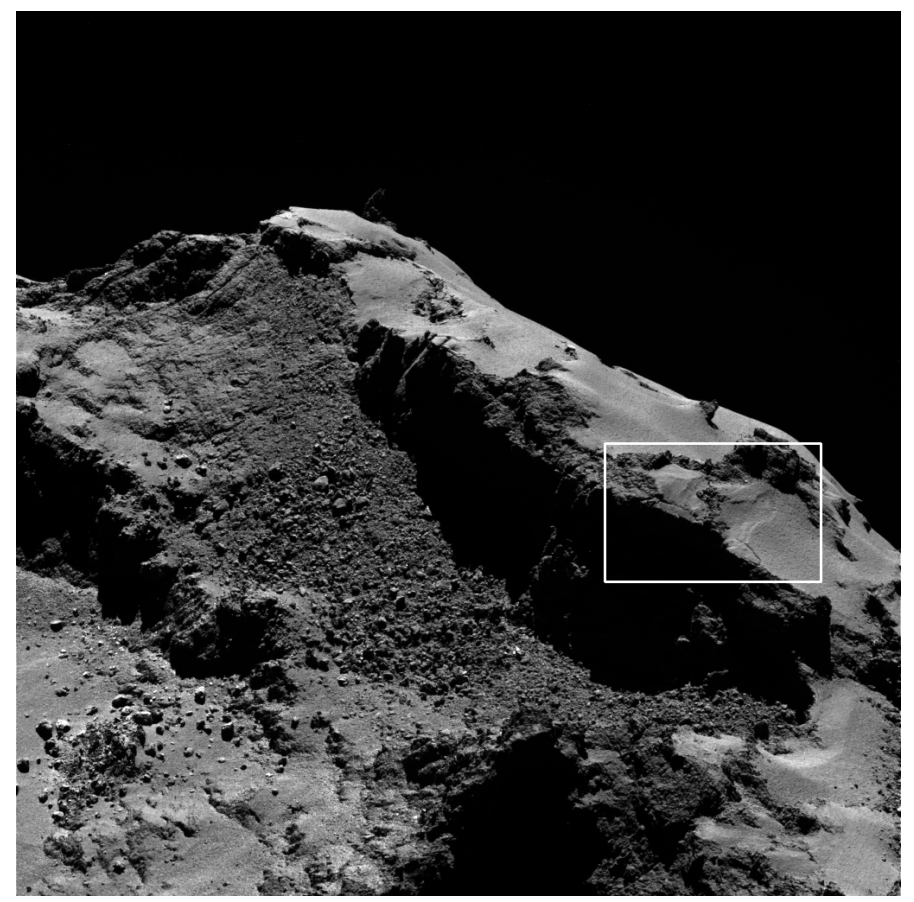

Fig. 4. Context image for Fig. 5 showing that the smooth deposit sits on a substrate. Evidence of substantial mass wasting are visible. Image: NAC_2014-10-01T04.36.23.549Z_ID10_1397549300_F22.

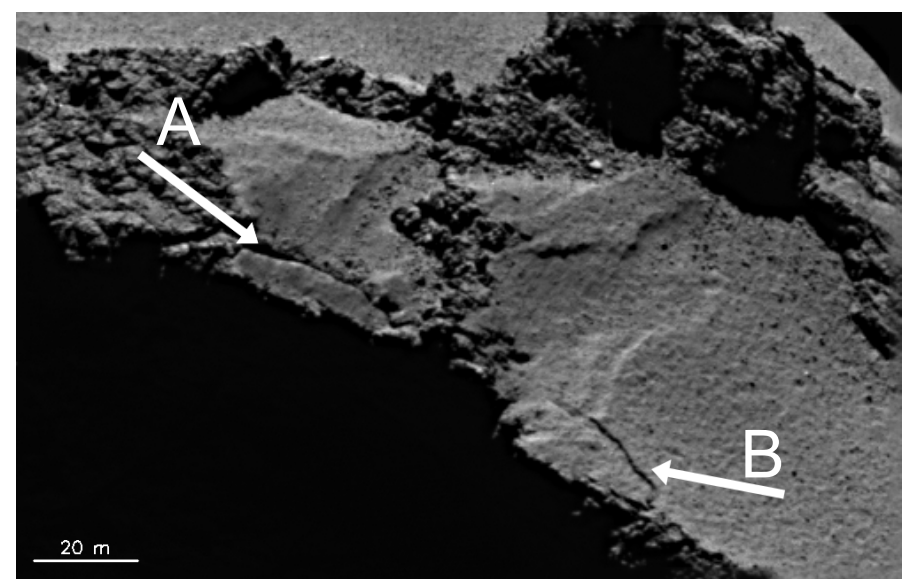

Fig. 5. Smooth deposit emplaced upon a substrate that has fractured (positions A and B) and collapsed at its edge. This fracturing occurred after most of the deposit was emplaced. Image: NAC_2014-1001T04.36.23.549Z_ID10_1397549300_F22.

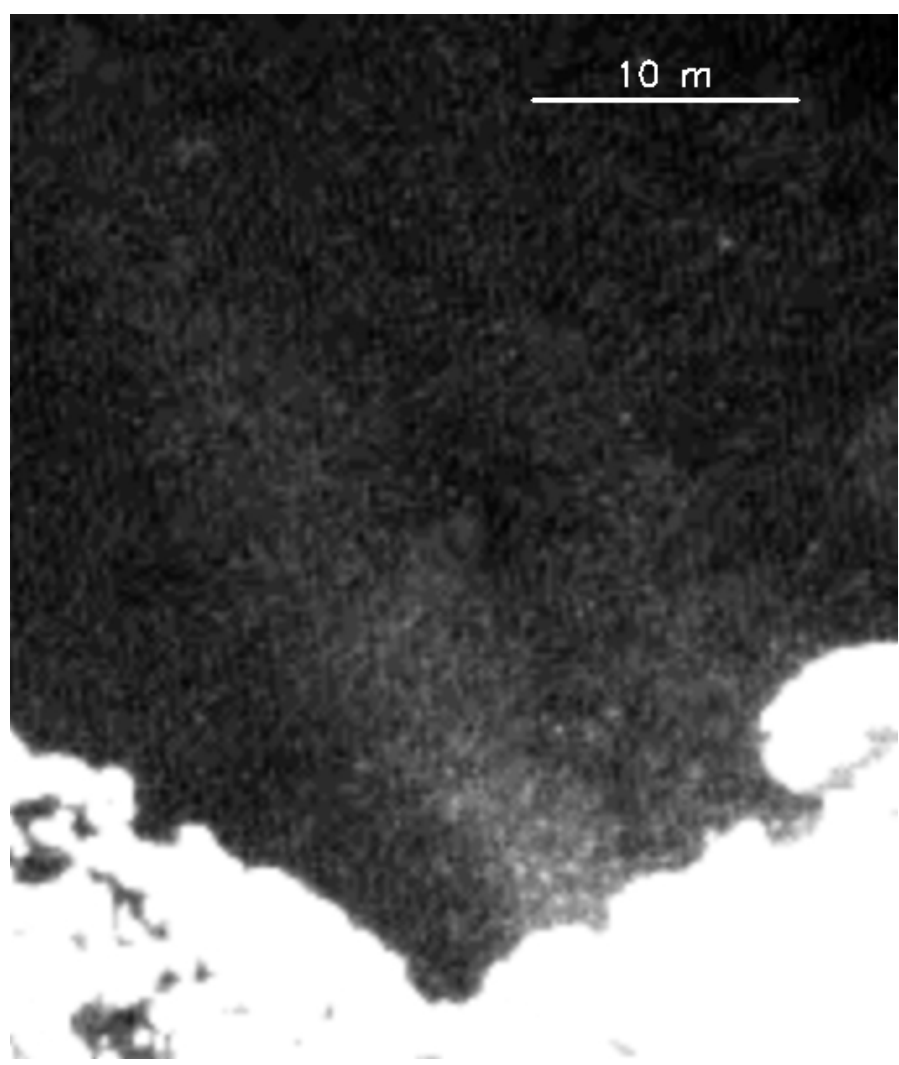

Fig. 6. Individual grains immediately above the surface shown against a shadowed area of the nucleus. Many individuals are not smeared at the resolution of the NAC. Given the exposure duration $(228 \mathrm{~ms})$ and the scale of the image ( $\approx 20 \mathrm{~cm} / \mathrm{px}$ at the particles), the in-plane velocity of these particles must be $<2 \mathrm{~m} / \mathrm{s}$. Image: NAC_2014-1014T21.20.32.331Z_ID10_1397549200_F22.

The exposure time is short $(228 \mathrm{~ms})$. However, the data were acquired with the spacecraft $10.69 \mathrm{~km}$ from the center of the nucleus, implying a spatial scale for the data here of $<20 \mathrm{~cm} / \mathrm{px}$. This also implies that smearing probably occurred for particles moving faster than $1 \mathrm{~m} / \mathrm{s}$ (i.e., particles close to or above escape velocity are probably smeared - many are not). The individual particles have brightnesses of about $2 \times 10^{-6} \mathrm{~W} \mathrm{~m}^{-2} \mathrm{sr}^{-1} \mathrm{~nm}^{-1}$ in the OSIRIS NAC orange filter $\left(\lambda_{\text {central }}=649 \mathrm{~nm}\right)$. The reflectance ratio between the particles and the adjacent illuminated surface is about 0.015 . Combining this with the pixel scale suggests that the particles are probably around $1 \mathrm{~cm}$ in radius. This is approximately equal to the maximum liftable size of particles for normal insolation at 3.2 AU on a low-albedo, water-icedominated surface in the absence of cohesive forces.

One might expect these particles to be accelerated once airborne. However, this acceleration is very slow for such large particles and may not be of long duration. Figure 6 shows that the jet itself is rather small $(\approx 20 \mathrm{~m}$ in diameter $)$. The commonly used equation for the drag force, $F_{\mathrm{D}}$, for a spherical particle of diameter, $d$, is

$F_{\mathrm{D}}=\frac{\pi d^{2}}{8} \rho C_{\mathrm{D}} v_{\mathrm{R}}^{2}$

where $C_{\mathrm{D}}$ is the drag coefficient, $v_{\mathrm{R}}$ is the relative velocity of the fluid with respect to the particle, and $\rho$ is the gas mass density. By dividing by the particle mass, we obtain an acceleration that is

$a_{\mathrm{d}}=\frac{\mathrm{d} v_{\mathrm{d}}}{\mathrm{d} t}=\frac{3}{4} \frac{\rho}{\rho_{\mathrm{d}}} C_{\mathrm{D}} \frac{v_{\mathrm{R}}^{2}}{d}$, to its source. A weaker source is also visible to its right. The jet is seen against the unilluminated nucleus, but is itself illuminated by the Sun. Individual particles can be seen in the outflow. 


$$
a_{\mathrm{d}}=\frac{\mathrm{d} v_{\mathrm{d}}}{\mathrm{d} t}=\frac{3}{4} \frac{Z M}{\rho_{\mathrm{d}}} C_{\mathrm{D}} \frac{v_{\mathrm{g}}}{d} .
$$

where $v_{\mathrm{d}}$ is the dust velocity arising from drag alone and $\rho_{\mathrm{d}}$ is the dust particle density. The gas density, $\rho$, can be replaced by $Z M / v_{\mathrm{g}}$, where $Z$ is the molecular flux, $v_{\mathrm{g}}$ is the gas velocity, and $M_{\mathrm{g}}$ is the gas molecular mass. If $v_{\mathrm{R}} \approx v_{\mathrm{g}}$, then

This acceleration is opposed by the gravitational acceleration, $g=G M / r^{2}$.

From these equations, the timescale needed for a particle to stay in a constant density and velocity flow to reach escape velocity can be written as

$t_{\mathrm{esc}} \approx \frac{\sqrt{2 G M / r}}{a_{\mathrm{d}}-\frac{G M}{r^{2}}}$,

assuming the distance moved in the time is smaller than the size of the nucleus. Even if one assumes now that the gas flux from an active source is that given by unrestricted free sublimation of water ice, then this time is on the order of minutes or longer for particles larger than a few hundred microns and realistic values for the other variables. It is already established, however, that the total gas production rate from the nucleus is on average around $1-5 \%$ of that expected for a water-ice comet of similar albedo (Snodgrass et al. 2013), which would increase $t_{\text {esc }}$ by factors of 20-100 unless the particles are being driven by a locally very high production rate spot on the nucleus. The size of the jet seen in Fig. 6, however, is small, and even if the particle is emitted from a locally high production rate spot, it will therefore enter a gas flow regime where densities (and hence accelerations) are potentially two orders of magnitude lower. Clearly, if this occurs before the particle has reached escape velocity, impact on a nucleus surface is a probable result.

The gas distribution in the inner coma provides little evidence for highly localized strong jets from pure water-ice surfaces (Bieler et al. 2015). In Sect. 3.2, we show calculations for an insolation-driven case that illustrate that significant numbers of large particles fall back even in the presence of gas outflow.

\subsection{Evidence of deposition of larger particles}

At the highest resolution, the smooth material is revealed to be inhomogeneous (Fig. 7) with significant variations in brightness. This suggests that the particle size in the deposit is large, which is consistent with a simple scenario where only the large dust particles are deposited because they are rapidly decoupled from the gas before reaching the extremely low escape velocity. The size-sorting produced by the coupling of dust particles to the gas naturally favors redeposition of only large particles. We note that the ROLIS observations from the Philae lander show a surface superposed by $\mathrm{cm}$-sized debris (Mottola et al. 2015).

Ejected small particles (i.e., micron-sized) are heated fairly rapidly once in sunlight (Lien 1990). However, the larger particles fail to equilibrate before re-impact, implying that they may retain substantial amounts of volatile material. In particular, icy material may be ejected and re-impact, producing bright spots on the surface. A possible example is shown in Fig. 7 in the lower left corner.

\subsection{Smooth surface formation scenarios}

There are several possible formation scenarios for the surface seen in Fig. 2. These include (1) deposition from a primarily vertical direction; (2) deposition on an originally flat surface with

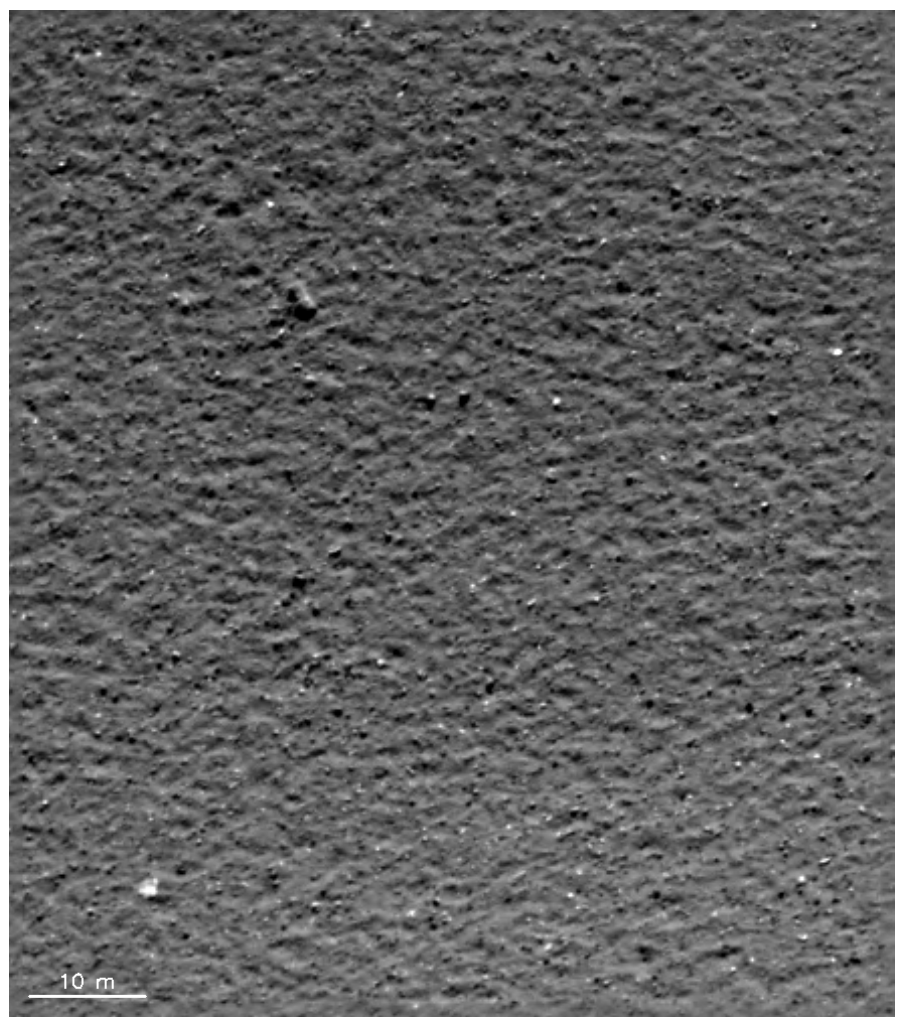

Fig. 7. High-resolution image of smooth terrain in the Ash region. The image scale is around $14 \mathrm{~cm} / \mathrm{px}$ (nominal scale $\approx 17 \mathrm{~cm} / \mathrm{px}$ when calculated with respect to the center of the nucleus). The phase angle is $91.7^{\circ}$. The appearance indicates heterogeneity in the $1-10 \mathrm{~cm}$ range. Image: NAC_2014-10-20T11.38.55.625Z_ID10_1397549400_F22.

subsequent pit formation through collapse, for example; (3) uniform deposition on the surface (a conformal coating) followed by preferential removal from vertical surfaces; (4) uniform deposition on the surface, but with no adherence of the depositing material to the vertical surfaces (for which mechanisms such as poor adherence and/or local outgassing could be envisaged); (5) surface processing (such as insolation weathering, particle impact) in situ to produce the observed smooth surface from material similar to the fractured material (thereby avoiding a deposition scenario). An airfall deposit seems most probable given that we have strong evidence for slow-moving, large particles close to the nucleus and that reduction in gas drag, at the edges of localized activity and when active regions shut down with the loss of insolation must occur (through the diurnal process for example). Furthermore, the absence of a deposit on vertical surfaces in several places on the nucleus (e.g., the Seth region) suggests that pit formation or collapse is not a universal explanation for clean vertical surfaces.

Referring back to Fig. 1, the regions that surround Ash, Babi, Ma'at, and Seth are essentially devoid of smooth material. Regions neighboring Ma' at on the head of the nucleus (Anuket and Maftet) do show some smooth material near their borders with Ma'at, but these are not dominant units in these regions. There is evidence of dune-like material in Maftet. Elsewhere, the transition from smooth material to consolidated material of a more rocky appearance is abrupt and usually associated with a topographic change. This is illustrated in Fig. 8. In this figure, Ash is at the top, the triangular flat surface (center right, marked A) is part of Apis, while Imhotep is to the lower left (and mostly in shadow here). Ash is covered with smooth material, but Apis 


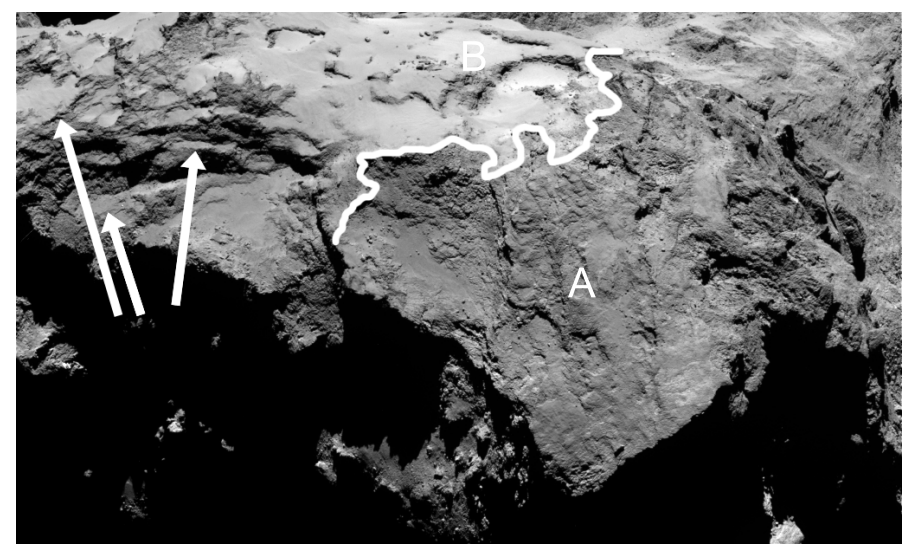

Fig. 8. View of the boundary of Apis and Ash. Ash (the area near B) shows smooth terrain with outcrops and exposures of more consolidated material beneath. Apis (area around A) appears rougher with less evidence of any airfall deposit. The region to the left of the figure appears layered. The arrows point to north-facing terraces and slopes that show evidence of the smooth material. Slopes nearly orthogonal to these surfaces are rough and show little evidence of the smooth deposit. The line defines part of the boundary between the smooth surfaces of Ash and the rougher terrains of Apis. Image:NAC_2014-0903T01.44.22.585Z_ID10_1397549900_F22. where $\boldsymbol{r}$ is the vector of point $\boldsymbol{y}$ to the volume element $\mathrm{d} V, G$ is the gravitational constant, and $\rho$ is the local density of the body.

The value of $\boldsymbol{a}_{G}$ was determined numerically. To do this, we discretized the volume with a resolution of $30 \mathrm{~m}$, resulting in 801757 volume elements $\Delta V$, and we assumed a constant density of $462 \mathrm{~kg} / \mathrm{m}^{3}$. The integral thus reduces to a sum over all these elements:

$\boldsymbol{a}_{G}(\boldsymbol{y})=G \rho \Delta V \sum_{n} \boldsymbol{r} /|\boldsymbol{r}|^{3}$.

This was done for more than 21 million points on a regular square Cartesian grid of $20 \mathrm{~km}$ dimension. Additionally, this calculation provides the local escape speed at the surface:

$v_{\mathrm{esc}}=\sqrt{2 r\left|\boldsymbol{a}_{G}\right|}$

which was found to agree with a surface gravitational acceleration computed using a more analytical method (Werner \& Scheeres 1997). (We note that a faster approximation to the Werner and Scheeres approach was presented by Cheng et al. 2002a).

Dust particles with a low initial speed at the surface can be tracked through the gravitational field. For this model we included Coriolis forces, but neglected the effects of gas drag - thus assuming that the dust grains have already decoupled from the gas flow near to the surface. (This is addressed in the next subsection.) The equation of motion to solve numerically is given by

$\frac{\mathrm{d} \boldsymbol{v}_{\mathrm{B}}}{\mathrm{d} t}=\boldsymbol{a}_{G}+\boldsymbol{a}_{\mathrm{C}}$,

where $a_{\mathrm{C}}=-2(\omega \times v)-\omega \times(\omega \times \boldsymbol{x})$ includes the Coriolis and centrifugal accelerations with the direction of $\omega$ being the rotation axis and its magnitude the angular speed of the nucleus' rotation. We assumed a pure spin.

We performed this calculation for particles originating from different regions (including the neck, the northern and the southern hemisphere), although we show here only the results for the neck. In each case, 100000 particles per initial speed were tracked though the gravity field with initial speeds ranging between $0.1 \mathrm{~m} / \mathrm{s}$ and $2 \mathrm{~m} / \mathrm{s}$ in steps of $0.1 \mathrm{~m} / \mathrm{s}$. The initial velocity vectors were randomly distributed within $3^{\circ}$ of the respective surface normal. Particles reaching a distance of $10 \mathrm{~km}$ of the nucleus center were assumed not to be ballistic and were not tracked further. This distance to the nucleus also corresponds to the outlet surface for calculations of the gas distribution to appear in Sects. 5 and 6.

The calculation for the neck produces results that are intuitively obvious. Figures 9 and 10 show the results for several velocities with only the facets from the neck used to generate the distribution. At low velocities, all particles re-impact the nucleus, as expected. It is apparent that at an ejection velocity of $0.7 \mathrm{~m} / \mathrm{s}$, re-impacting particles cover the northern hemisphere of the nucleus, with relatively few reaching the southern hemisphere, the Khepry region, or Imhotep. Calculations at lower velocities show (as might be expected) that the extent of the deposition over the northern hemisphere is reduced, with particles failing to escape from the neck unless their velocities are $>0.5 \mathrm{~m} / \mathrm{s}$. Higher velocities lead to escape $(50 \%$ of particles ejected at $1.0 \mathrm{~m} / \mathrm{s}$ escape), and deposition on the southern hemisphere occurs, providing global deposition but lower numbers of impacts per unit surface area. This illustrates that we have a type of velocity filtering by the form of the nucleus in combination with emission from the neck. While particles may be ejected 

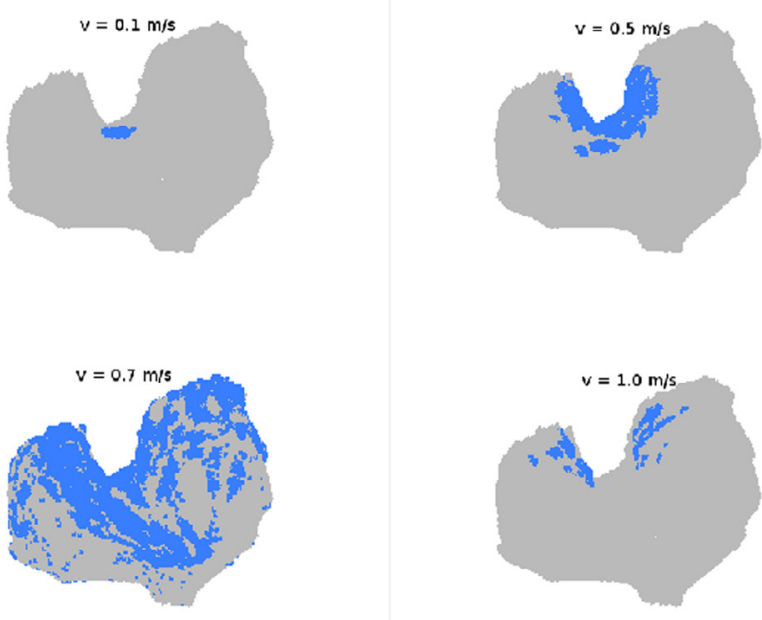

Fig. 9. Positions of re-impacting particles emitted from the neck for different ejection velocities. Side view showing that ejection speeds of $<0.5 \mathrm{~m} / \mathrm{s}$ fail to exit the neck, while ejection speeds $>1.0 \mathrm{~m} / \mathrm{s}$ are sufficient to exit the domain except for those particles that collide with the surfaces of Seth and Hapi before they escape.
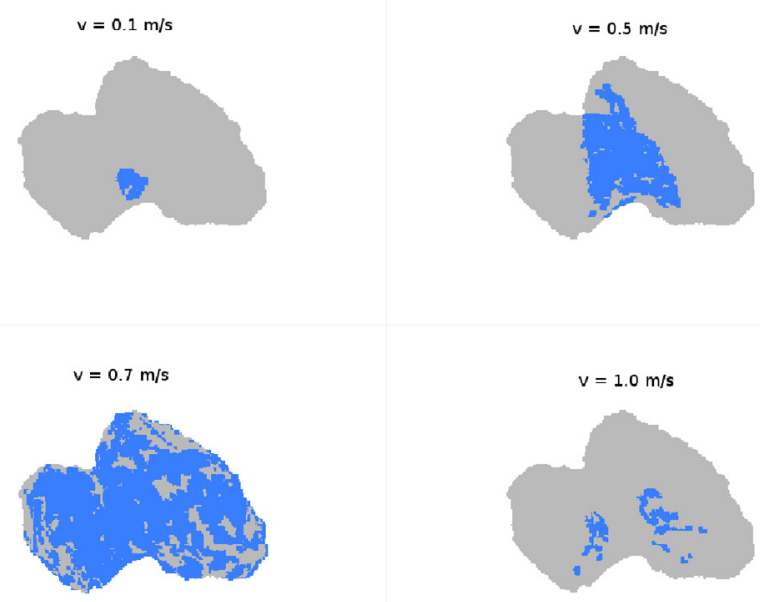

Fig. 10. As Fig. 9, but viewed from above the north pole, illustrating the extensive coverage of the northern hemisphere of the nucleus for ejection speeds of about $0.7 \mathrm{~m} / \mathrm{s}$.

from the neck over a range of velocities, only those in the range 20.5 to $0.9 \mathrm{~m} / \mathrm{s}$ re-impact the nucleus outside the neck region, and 3 particles with initial speeds in the range 0.5 to $0.8 \mathrm{~m} / \mathrm{s}$ build up 4 the highest surface depths on the northern hemisphere. This il5 lustrates that the concept of airfall as a production mechanism 6 for the smooth material on Ash, Ma'at, and Babi is plausible.

\section{3.2. Influence of gas drag}

8 A key assumption in the previous section is that particles in9 volved in the airfall process are too large to be significantly af10 fected by gas drag. To illustrate that this is plausible, we ran a 11 simulation using a 3D direct simulation Monte Carlo (DSMC) 12 code with an unstructured grid known as $\mathrm{PDSC}^{++}$. This code 13 has been used previously to model the water vapor distribution 14 in the vicinity of comet 9P/Tempel 1 (Finklenburg et al. 2014). and co-workers (Wu \& Lian 2003; Wu \& Tseng 2005; Wu et al. 2004). PDSC ${ }^{++}$allows a simulation of 2D, 2D-axisymmetric, and $3 \mathrm{D}$ flows on hybrid unstructured grids. The code was parallelized, allowing a much larger number of cells, and was implemented on several clusters in Bern and Taiwan. The code is especially useful in that it is able to treat the high-density gradients by implementation of a variable time-step and a transient adaptive subcell technique to increase computational speed and accuracy in the regions of high density (Finklenburg et al. 2014). The implementation of the code specifically for 67P, a sensitivity study with respect to input parameters used for cometary studies, and a more detailed evaluation of the results of application to $67 \mathrm{P}$ will be presented in future publications.

We used here an SPC shape model of the nucleus with 25796 facets. A simple thermal model was constructed omitting thermal conductivity (i.e., the thermal inertia was set to zero), but including sublimation of water ice. The sublimation coefficient was set to 1 for simplicity. The thermal balance was produced by

$0=\frac{S\left(1-A_{\mathrm{H}}\right) \cos \iota}{R_{\mathrm{h}}{ }^{2}}-\epsilon \sigma T^{4}-L \frac{\mathrm{d} m}{\mathrm{~d} t}$,

where $A_{\mathrm{H}}$ is the directional-hemispheric albedo (set to 0.04 ), $\mathrm{S}$ is the solar constant at $1 \mathrm{AU}, \iota$ is the angle of incidence, $R_{\mathrm{h}}$ is the heliocentric distance of the comet, $\epsilon$ is the IR emissivity (set to $0.9), \sigma$ is the Stefan-Boltzmann constant, $L$ is the latent heat of sublimation of water ice, and $\mathrm{d} m / \mathrm{d} t$ is the sublimation rate.

The sublimation rate was computed from the surface temperature, $T$, using the equation

$\frac{\mathrm{d} m}{\mathrm{~d} t}=p_{\operatorname{evp}} \sqrt{\frac{M_{\mathrm{H}_{2} \mathrm{O}}}{2 \pi k T}}$,

where the equilibrium vapor pressure of water vapor $\left(p_{\text {evp }}\right)$ was computed from values given by Huebner et al. (2006). This scheme provided a sublimation flux and a gas temperature for each facet. For unilluminated surfaces, the gas flux was set to zero and the nominal surface temperature to $1 \mathrm{~K}$.

Use of this scheme would normally produce gas production rates far in excess of what is observed. Hence, we scaled the fluxes from each facet to produce production rates that are closer to those observed at 67P. One can visualize this as being equivalent to only a fraction of the surface facet being active, with the rest being inert.

For this calculation, we used a homogeneous model where sublimation is only driven by insolation, following the conclusions of Bieler et al. (2015). Equation (1) was then used with a test particle approach (Crifo et al. 2005) to compare the percentage of particles that can be lifted by the gas flow (in the absence of cohesive forces) with the number of particles that escape the gravitational field of the nucleus. The number of particles entering the system was set to be directly proportional to the gas production rate at each facet. The particles were split into 53 size bins from 0.1 micron to 3 millimeters in radius. The computation was made for the comet at $3.4 \mathrm{AU}$ with a total gas production rate of $1.55 \mathrm{~kg} / \mathrm{s}$ and for only one orientation of the nucleus as a proof of concept. The calculation was run in steady-state (i.e., no nucleus rotation or Coriolis force) and with a point-source gravity model. (The full coupling of the gas model with the true gravity field and rotation remains to be completed at this stage.) The result is shown in Fig. 11.

Interestingly, a small fraction of even very small particles are not lifted. These particles come from facets where the gas production is very weak as a result of very oblique insolation. Furthermore, a significant fraction of small particles, although 


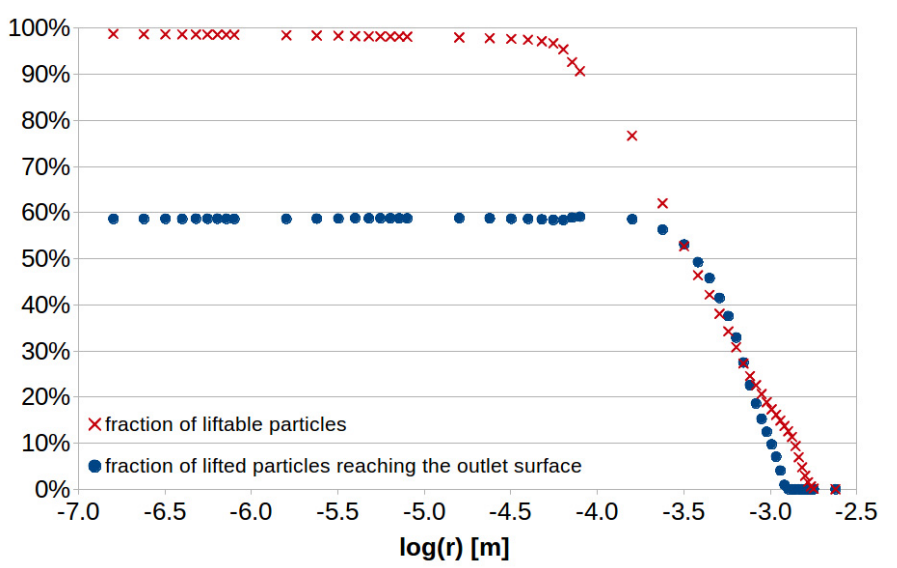

Fig. 11. Red crosses: the fraction of particles lifted in a homogeneous DSMC gas outflow model with surface particle densities proportional to the local gas production rate expressed as a function of the log of the particle radius. No surface cohesive forces are included. Blue dots: the fraction of the lifted particles that then reach the outlet surface, $10 \mathrm{~km}$ from the center of the nucleus. The plot shows that particles ejected from the surface larger than about $1.3 \mathrm{~mm}$ are not accelerated by gas drag to beyond escape velocity. to keep the active areas within Hapi clear of the returning material, such that only weakly or inactive areas receive a returning deposit.

\subsection{Gas emission from icy boulders}

Although the process by which larger grains are ejected from the surface of $67 \mathrm{P}$ is still highly uncertain, it seems reasonable to assume that ejected grains and chunks can be both non-volatile and volatile. A'Hearn et al. (2011) have argued that icy chunks in the size range of 15 to $20 \mathrm{~cm}$ have been ejected from the surface of $103 \mathrm{P} /$ Hartley 2 with some at velocities below the local escape velocity. Hence, there is already evidence that volatile-bearing large grains or chunks can be lifted from a cometary nucleus into orbits and re-impact the nucleus far from the original source. The ejection of larger icy chunks may lead to low-velocity impact far away from the source, and indeed in regions where the insolation would be insufficient to sublime the ice in the short term (e.g., up to half a comet orbital period if the chunks impact near the unilluminated pole). A key consequence of this is that icy chunks can become distributed low-level gas sources over a significant fraction of the nucleus as a result of airfall. The nucleus is then not homogeneously outgassing in response to the insolation, but neither are active areas exclusive sources of gas. Given that there are differences in the source mechanism, it would therefore be expected if properties such as the dust-togas production rate ratio would be affected. The emission of icy chunks from active regions alone produces ambiguity in the definition of the dust-to-gas ratio but, in addition, the sublimation of the chunks present in the airfall deposit may provide locally low values.

\subsection{Residues}

An important aspect of the airfall deposit is that once any residual water ice has been removed, the residue is likely to contain a substantial organic component. Composition analyses of dust at comet $1 \mathrm{P} /$ Halley have shown the relative importance of organics with respect to silicate particles (Jessberger et al. 1988). Similarly, ground-based infrared spectroscopy has consistently shown evidence for a $3.4 \mu \mathrm{m}$ absorption diagnostic of the C-H stretch, while VIRTIS observations of the nucleus of $67 \mathrm{P}$ have already revealed a broad absorption band at this wavelength (Capaccioni et al. 2015). Observations of a blue surface (negative spectral slope with wavelength) in the extreme ultraviolet wavelengths with the ALICE spectrometer have also been interpreted in terms of a tholin-type surface composition (S.A. Stern, pers. comm., presentation at DPS 2014).

Recent laboratory work has indicated that organic residues from sublimation of an ice-tholin mixture can rapidly combine to produce fluffy particles that are much larger than the original constituents (Poch et al. 2015). This occurs even if the original organic particles are separated from each other by encapsulation in the ice. Figure 12 shows the result of such a sublimation experiment in the SCITEAS chamber (Pommerol et al. 2015) at the University of Bern. An intermixture of $0.1 \%$ tholins (in particulate form with a size of $315 \pm 185 \mathrm{~nm}$ Carrasco et al. 2009) and water-ice particles of around $70 \mu \mathrm{m}$ in diameter were evolved in the SCITEAS chamber. Sublimation of the water ice was allowed to occur for $12.5 \mathrm{~h}$ at $\approx 10^{-5}$ mbar and $200-220 \mathrm{~K}$. A coherent, porous, water-free mantle of tholins is produced by this process. The tholin particles combine readily in this environment, producing larger structures. Centimeter-sized fragments 


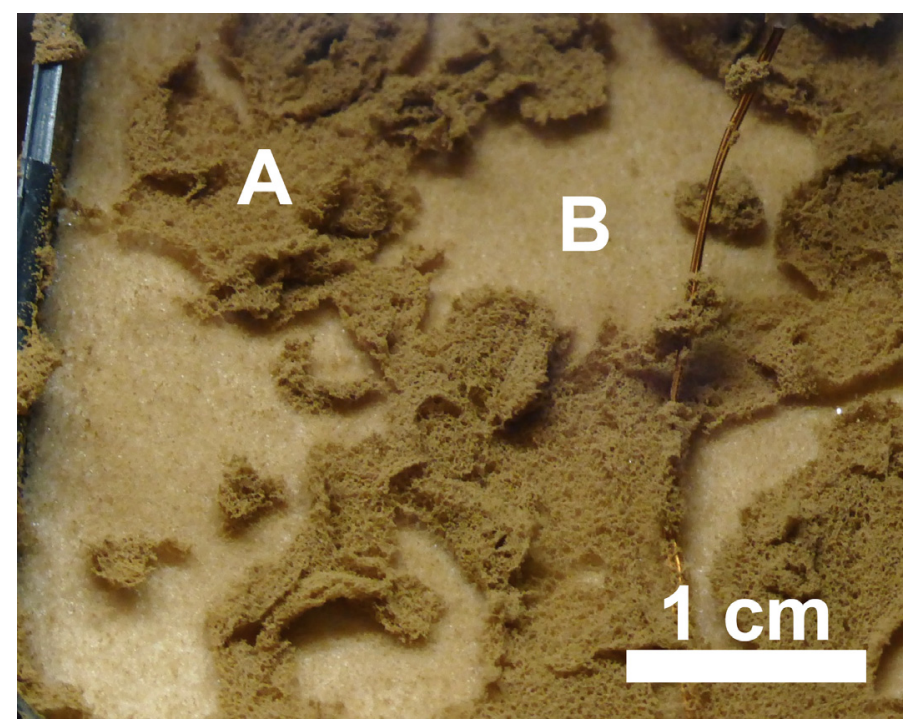

Fig. 12. Picture of a mantle of tholins obtained after sublimation of an intermixture of $0.1 \%$ tholins and water ice particles of around $70 \mu \mathrm{m}$. The sublimation was performed during $12.5 \mathrm{~h}$ at $\approx 10^{-5}$ mbar and 200-220 K in the SCITEAS simulation chamber. A: coherent porous mantle made of water-free tholins; B: bright area made of water-ice particles exposed to the surface after ejection of a cm-sized fragment of the mantle. This image was taken in situ, while the sample was sublimating inside the SCITEAS chamber. of the mantle are occasionally ejected by the sublimation process as it proceeds. Even if the tholins are isolated by encapsulating them in an ice shell before initiating sublimation (a socalled intramixture), similar types of structures form (Poch et al. 2015). Hence, the formation of a surface organic matrix through sublimation of an ice-organic mixture on the smooth terrain is plausible.

\subsection{Thermal inertia}

The airfall results in a very slow collision with the surface. Typical velocities are lower than $1 \mathrm{~m} / \mathrm{s}$ (i.e., lower than the escape velocity). With such low-velocity collisions, we expect the build-up of a fluffy deposit that is both porous and compressible. Given that the contact area between particles is then likely to be very small, this would lead to a low thermal conductivity and hence low thermal inertia. Low values of thermal inertia for cometary surfaces have been inferred through surface temperature measurements for many years, starting with Emerich et al. (1987). Low thermal inertia $\left(<70 \mathrm{~J} \mathrm{~m}^{-2} \mathrm{~K}^{-1} \mathrm{~s}^{-1 / 2}\right)$ was also noted for comet 9P/Tempel 1 (Groussin et al. 2013). Latest results from the MIRO experiment on Rosetta suggest that this is also true for 67P (Gulkis et al. 2015).

A possible inconsistency in this conclusion is that Kömle et al. (1996) measured the thermal conductivity of organic residues and concluded that the conductivity was at least an order of magnitude higher than the typical value for a loose dust mantle containing no organic material, although the sample production process was markedly different from the airfall process suggested here. The conductivity in the measurements of Kömle et al. also showed a depth dependence, which may indicate that the deposition rate can influence the bulk conductivity of the material. For the case of 67P, this may lead to a variable thermal conductivity over the nucleus depending upon the local airfall deposition rate. We note that Davidsson et al. (2013) found that the thermal inertia of $9 \mathrm{P} /$ Tempel 1 , as inferred by analyzing the near-infrared emission measured by Deep Impact, using thermophysical models that included surface roughness as well as heat conduction, varied across the surface.

\subsection{Changes in surface properties with depth}

The build-up of a fluffy deposit that is both porous and compressible has significant implications for the interpretation of the results from the Philae lander. The imprint made by the first impact of the lander with the surface is consistent with a $10-20 \mathrm{~cm}$ compression of the surface layer (e.g., Heggy et al., in prep.). This, however, may only be indicative of the compressive strength of the fluffy deposit and not of the bulk of the comet below. Hence, a low compressive strength surface layer with a higher strength subsurface structure would be consistent with this model.

\section{Surface ventifacts (ripples, moats, and wind-tails)}

\subsection{Introduction}

The effects of extreme pressure gradients on loose surface material on comets has rarely been explored. Kührt \& Keller (1994) pointed out the importance of cohesive forces and showed that over a wide parameter range, pressure gradients in a numerically modeled cometary crust would be insufficient to exceed them. Cheng et al. (2013) appears to have been the first to consider erosion driven by cometary outgassing using formulations similar to those used to study saltation on Mars (Greeley \& Iversen 1985) and, following Scheeres et al. (2010), also noted the importance of cohesive forces between particles on bodies with low surface gravity. The OSIRIS observations suggest that these ideas are of considerable importance.

\subsection{Observations of ripples}

When a gas flux over an immobile bed of cohesionless grains becomes sufficiently high, the grains are set in motion and dunes form. The surfaces of aeolian sand dunes are not smooth, but are usually in the form of regular patterns (ripples), transverse to the wind direction. Mature ripples are asymmetrical in cross section. Their stoss (upwind) slopes are typically much fainter than the shorter lee (downwind) slopes. The steepness of the lee slopes cannot exceed and usually does not reach the angle of repose. The ripples have convex stoss slopes, concave lee slopes, and flattened crests (Prigozhin 1999).

In Fig. 13 we show what appear to be aeolian ripples in the Hapi region (Thomas et al. 2015) on 67P. This image was acquired on 17 Sept. 2014 with the NAC from a cometocentric distance of $28.8 \mathrm{~km}$ when the comet was $3.346 \mathrm{AU}$ from the Sun. The phase angle is 85.9 degrees, with the projection of the vector to the Sun being vertically upward on the image. The scale of the image is $0.54 \mathrm{~m} / \mathrm{px}$ when computed for the center of the nucleus. The ripples are roughly aligned, and one can estimate a wavelength by counting the number of crests along a line orthogonal to the aligned ripples. This gives a value of $5.50 \mathrm{~m}$ in the image plane averaged over 11 crests. We observe the ripples obliquely, and hence there is a foreshortening effect. By using the 3D shape model of the nucleus, we can measure the distance, which leads to a wavelength of $12.1 \mathrm{~m}$. The observer in Fig. 13 views the surface of the ripples at an elevation of $\approx 27^{\circ}$. Another image (NAC_2014-09-02T21.44.22.575Z_ID10_1397549800_F22) at lower resolution, but at a more favorable viewing angle for direct 


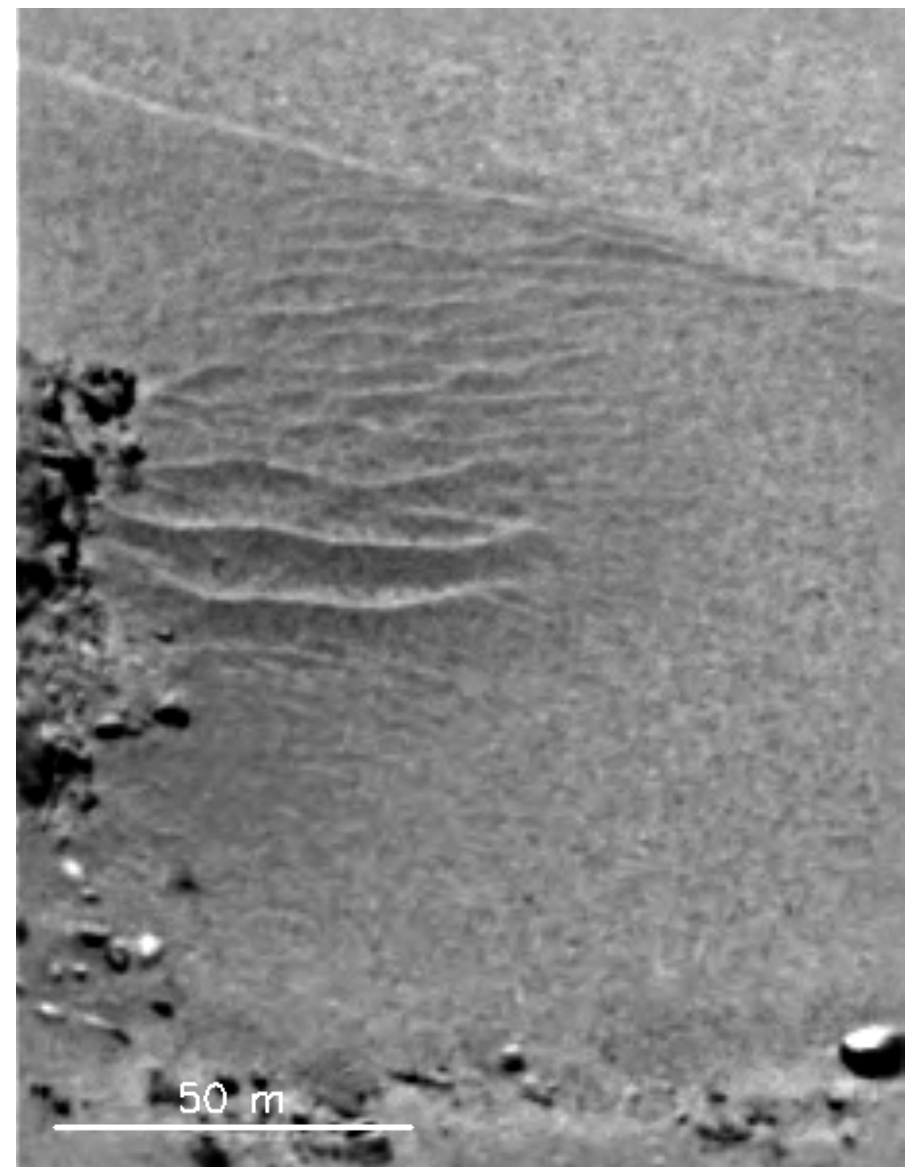

Fig. 13. Aeolian ripples in the Hapi region on 67P. Image NAC_201409-17T23.52.43.330Z_ID10_1397549400_F22. measurement, places a lower limit of $117.7 \mathrm{~m}$ (a wavelength $>10.7 \mathrm{~m}$ ) for the length of the ripple field (Fig. 14).

A major source of error arises from the estimate of the number of crests. We identified 11 clear crests in the central section of the ripple field. However, by selecting a specific path crossing bifurcated ripples, a maximum of 13 crests can be reached. Hence, the wavelength may be up to $25 \%$ shorter. The width of the ripple field is around $60 \mathrm{~m}$.

The shape models of the nucleus appear to be of just sufficient accuracy to determine the amplitudes of the two largest ripples. We chose to use the stereo photoclinometry (SPC; Gaskell et al. 2008) model for this purpose. The SPC and stereo photogrammetry SPG) techniques are complementary for stereo reconstruction. SPG (Preusker et al. 2012) is optimum when relief is significant, but SPC is more useful when the surface is relatively smooth, as is the case with the ripple field.

We smeasured the peak-to-valley amplitudes of the two most apparent ripples in the shape model. To acquire a statistics, the measurements were made at eight different positions separated by 3-4 $\mathrm{m}$ along each ripple. We obtained values of $22 \pm 12 \mathrm{~cm}$ and $26 \pm 14 \mathrm{~cm}$ for the two ripples. The SPC model tends to underestimate the amplitude, and values around a factor of 2 higher would probably still be consistent with the data, which implies a ratio of ripple amplitude to wavelength of $(A / \lambda)$ of 0.02-0.04. In Earth-based conditions, $A / \lambda$ is roughly constant at 0.04 , and hence our observations are reasonably consistent with what might be expected.

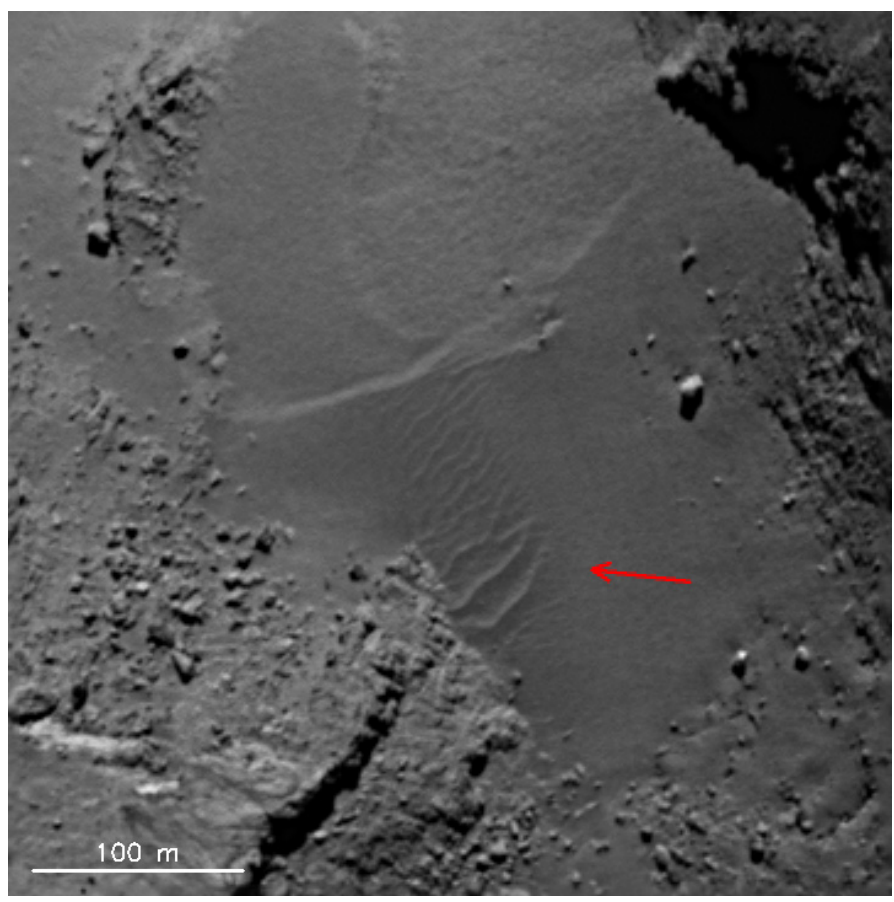

Fig. 14. Aeolian ripples in the Hapi region on $67 \mathrm{P}$ seen at a viewing geometry more orthogonal to the surface. Image NAC_2014-0902T21.44.22.575Z_ID10_1397549800_F22.

\subsection{Observations of dune-like structures and putative wind-tails}

The possible presence of dune-like structures in the Maftet region of 67P was discussed in Thomas et al. (2015). Most of these structures are close to the original Philae landing site and have been mapped by La Forgia et al. (2015) in their characterization of the site. They showed a preferential orientation and suggested that the structures might be related to longitudinal dunes.

Figure 15 shows part of the Maftet region (to the right), but also includes the Nut depression (marked A in Fig. 15) and the flat surface of Serqet (marked B). Serqet is remarkable because of a ridge of consolidated material that separates Serqet, Nut, and Ash from the lower lying regions of Anuket and Hathor. Serqet also contains a flat and smooth plain (roughly $280 \mathrm{~m} \times 850 \mathrm{~m}$ in dimension) that appears to be dust covered (El-Maarry et al. 2015). Figure 15 shows that the smooth plain has irregular ripples across its surface. The Nut region is covered with boulders with consolidated and fractured material below. At positions $\mathrm{C}$, $\mathrm{D}$, and $\mathrm{E}$ in Fig. 15, smooth material is visible. This material lies on the more consolidated material; the surfaces in the vicinity of position E illustrate this well. Figure 16 shows another excellent example. At position A in Fig. 16, the smooth material clearly lies on fractured consolidated material. The shadows also indicate that the dune material has a positive relief with respect to the underlying fractured material. Figure 15 also shows in positions $\mathrm{F}$ and $\mathrm{G}$ (and possibly $\mathrm{H}$ ) that some boulders are on or in the smooth material and that small tails have built up to one side. This type of arrangement (smooth material to one side of the boulder and slightly topographically higher than the surroundings) is observed elsewhere on the nucleus as well. In Fig. 17, for example, which is part of an image acquired in the Hapi region, smoother material is seen to one side of the boulders.

The entire Maftet region gives the impression that the smooth material has been mobile. Using the SPC shape model, we estimated the height of the dune-like structure at position D 


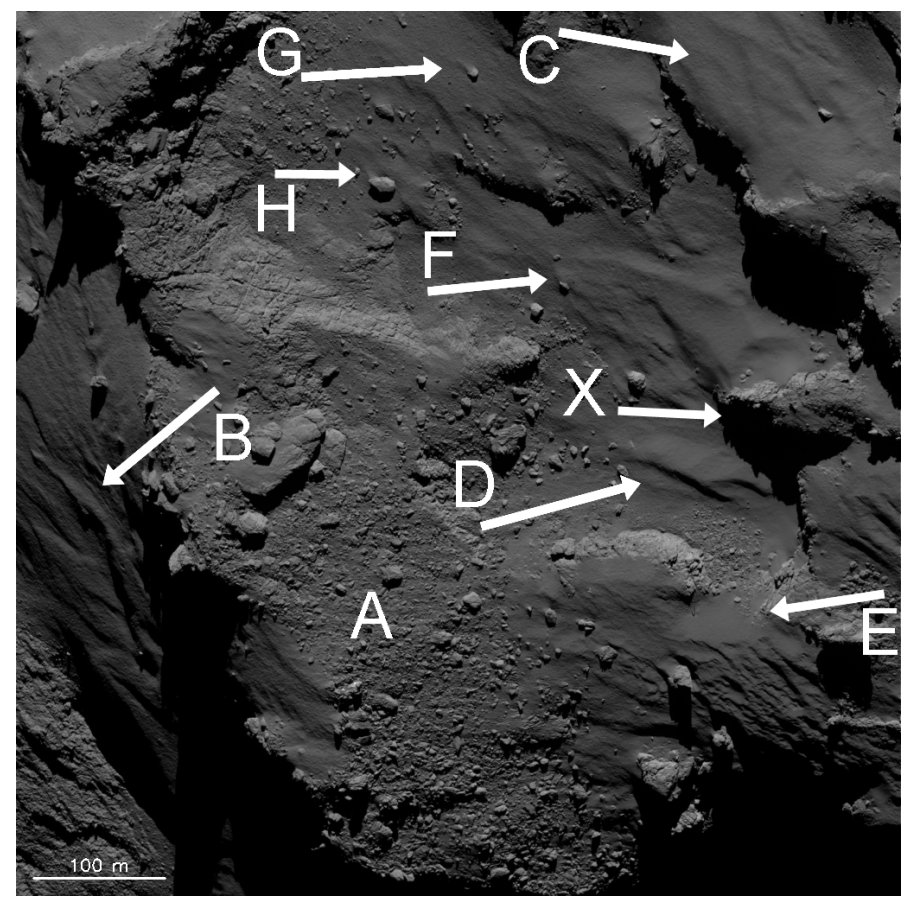

Fig. 15. Position A marks the centre of the Nut region on the nucleus. To the left is the Serqet region. Smooth ripple structures can be seen in this flat surface of Serqet (marked B). Ripples and dunelike structures are also seen in the Maftet region (which is to the right and below Nut) at positions $\mathrm{C}, \mathrm{D}$, and $\mathrm{E}$. The smooth material appears lie on a fractured, more consolidated base. Some boulders (e.g. G and H) appear to have wind-tails. Image: NAC_2014-1112T15.13.51.581Z_ID10_1397549200_F22.

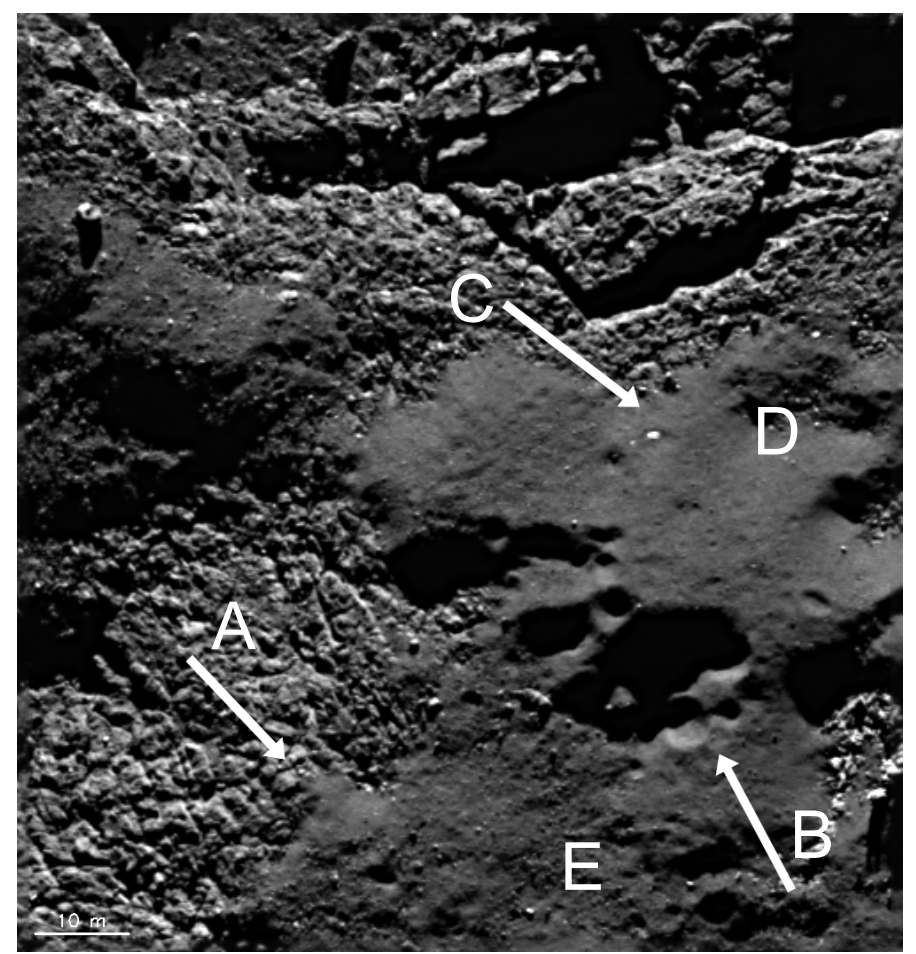

Fig. 16. Another part of the Maftet region showing that the smooth material lies on top of the fractured surface (see position A). There are also pits in the dune-like material (B) but without a preferred orientation. Moreover (position $\mathrm{C}$ ), the smooth material is inhomogeneous at high resolution with brighter spot material evident. Image: NAC_201410-19T13.09.06.551Z_ID10_1397549600_F22.

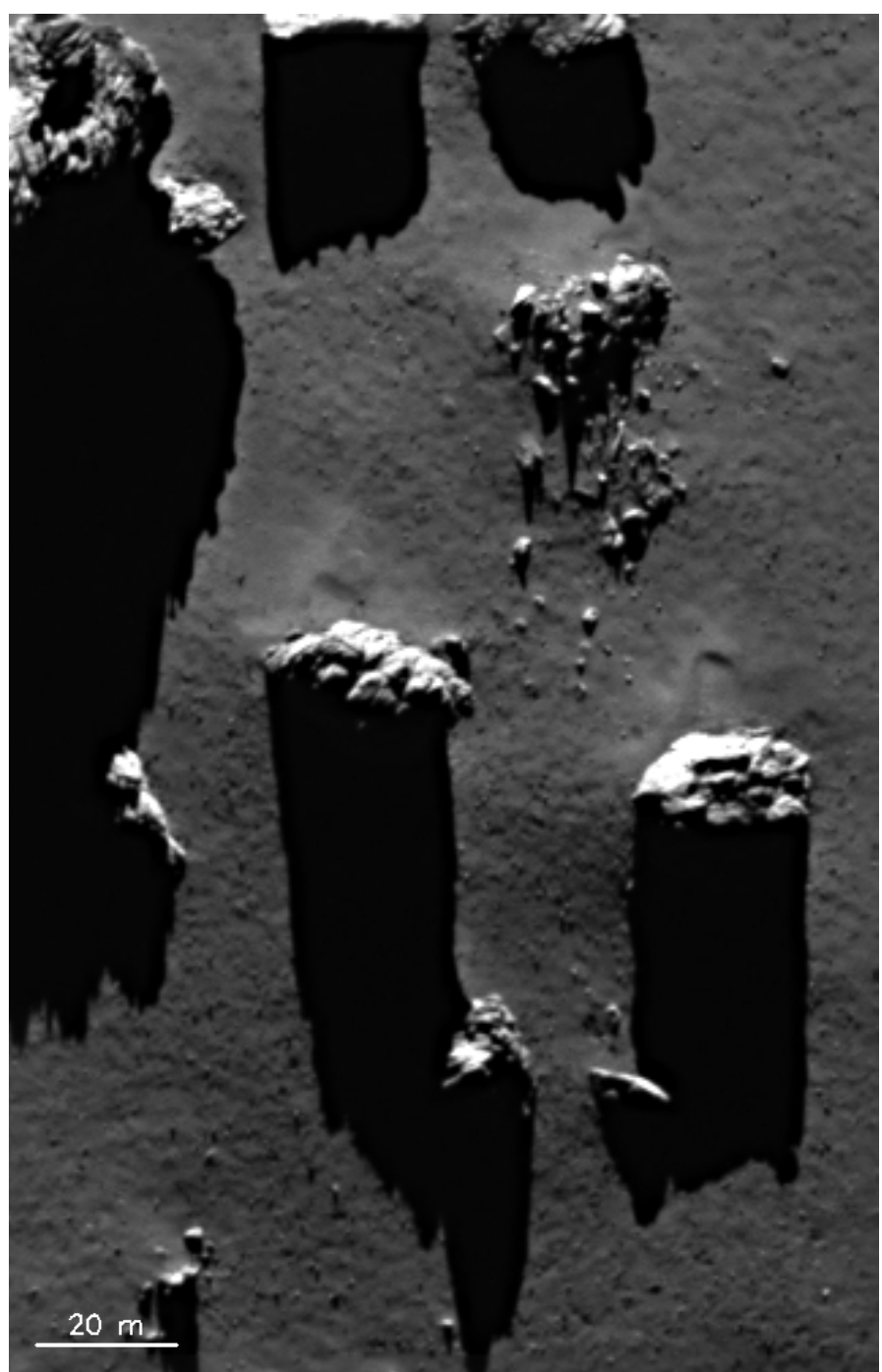

Fig. 17. Putative wind-tails in the Hapi region. Smooth, finer material preferentially lies at the upper side of the boulders in the view. Image: NAC_2014-12-10T06.28.55.791Z_ID10_1397549000_F22.

in Fig. 15 to be between 1.5 and $2.5 \mathrm{~m}$, and hence these are not substantial formations. Most of the structures identified by La Forgia et al. (2015) are not evident in the most recent SPC shape model, for example. If the structure at position $\mathrm{D}$ is a dune, then the slip-face appears to be facing the Nut-Maftet boundary, suggesting gas flow from the Ma'at region.

We have shown that airfall has produced meter-thick deposits. Where this occurs, the nucleus activity is likely to be reduced or choked entirely. Since we also see dust emission from the Ma'at region, it seems probable that the observed outcrops of weakly consolidated material are more active. The dune-like formation at position $\mathrm{D}$ is within a few meters of an outcrop.

Remarkably, the smooth material is pitted in some areas. Examples are shown in Fig. 18. At position A, a dune-like slope of smooth material is visible. It is striking, however, that the smooth material appears eroded and pitted (e.g., at position B). In some local areas, the pits appear to be aligned in a preferred orientation. Evidence for this is shown in Fig. 18 (although there are better examples elsewhere). This is not, however, a universal property of the dune-pit structures. Figure 16 shows a pit cluster (position B), and there are isolated pits elsewhere in the field. 


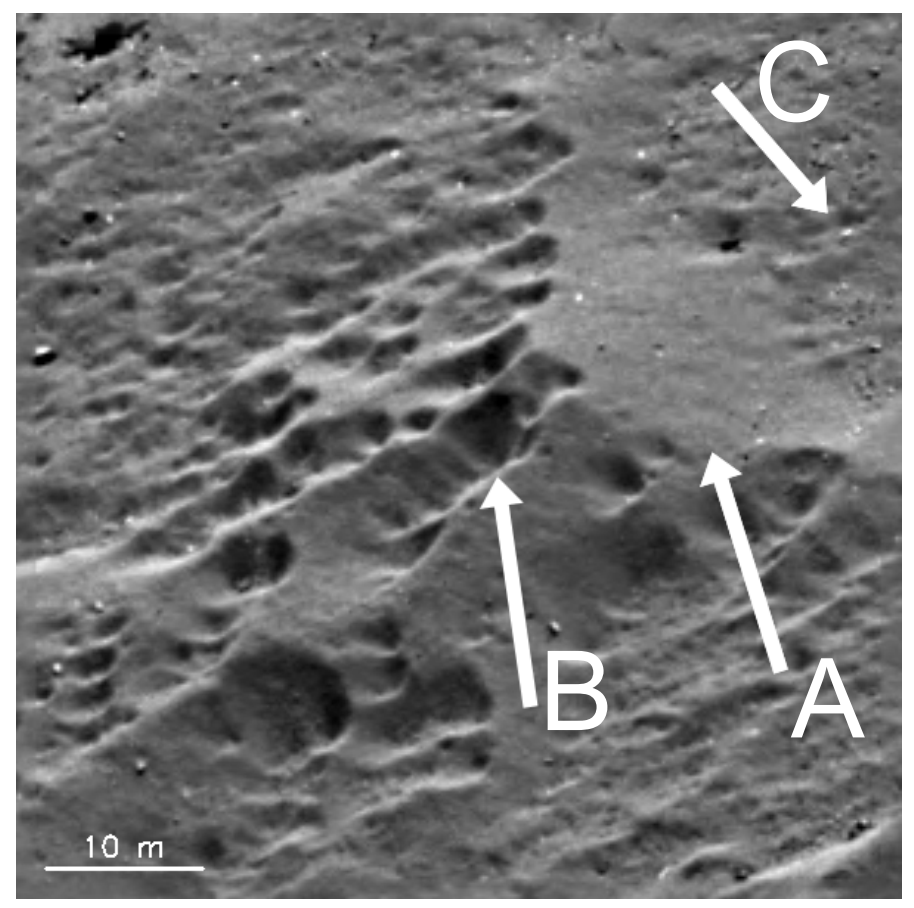

Fig. 18. High-resolution image of a putative dune in Maftet. Position A shows a smooth surface. To the left (position B), pits have formed in the smooth material. The pits are aligned. The bright spots in the smooth material (also position $\mathrm{C}$ ) may be volatile-rich chunks. Image: NAC_2014-10-19T12.22.15.525Z_ID10_1397549600_F22.

We have referred throughout the above to the dune-like structures as being composed of "smooth" material. However, at the highest resolution, it is apparent that the material is heterogeneous and, in some areas, rough at submeter scales. The wind5 tails seem to be somewhat smoother, possibly indicating smaller particle sizes. Bright spots of material are visible, which we in7 fer to be similar to the bright spots seen in the smooth material on the surface of Ash (e.g., in Fig. 7). Figure 18 shows an example at position C. Furthermore, a detailed comparison of the terrain near positions D and E in Fig. 16 shows that there are major differences in the small-scale (submeter) roughness.

Although the production mechanism is unknown, it seems highly unlikely that the smooth surface was formed with a pitted appearance. It has evolved to produce this appearance. The differences in surface roughness evident in some places also suggest post-depositional modification.

The latitudes of the pitted dune-like terrains are close to the highest northern latitude of the Sun on the comet's surface and, qualitatively, the southern faces are affected by pitting. Dunes at higher latitudes show no pits. Hence insolation must be a candidate for the pit-production mechanism.

\subsection{Wind-driven saltation}

Wind-blown particles on Earth or on Mars can include particles transported in suspension, by saltation, and/or by creep/reptation mechanisms (Greeley et al. 2002). The particle sizes involved in each mechanism are different, with those involved in creep and reptation being the largest (e.g., Kok et al. 2012). The production of aeolian ripples appears quite straightforward at first glance. When "saltons" (high-energy grains) collide with the bed, they eject grains of smaller energy, "reptons". The windward slope of a small bump is submitted to more impacts than the lee slope, so that the flux of reptons is higher uphill than downhill, and thereby the height of the crest is amplified (Andreotti et al. 2006). The created pattern, however, tends to saturate such that a state is reached where the ripples essentially propagate without changing shape and amplitude anymore. Andreotti et al. (2006) showed using an initially corrugated bed that the ripple pattern converges toward different stable nonlinear solutions, depending upon the initial conditions. As pointed out by Greeley et al. (2002), the critical factor in ripple formation is the reptation-creep length and not the saltation length. The data from Andreotti et al. (2006) suggest that

$\frac{\lambda}{d}=K \frac{u^{*}}{u_{\mathrm{th}}}$

where $\lambda$ is the final ripple wavelength, $d$ is the grain size (diameter), $u^{*}$ is the wind shear velocity, $u_{\text {th }}$ is the shear velocity at the fluid threshold, and $K$ is a proportionality constant that was suggested to be dependent on the density ratio $\rho_{\mathrm{s}} / \rho_{\mathrm{g}}\left(\rho_{\mathrm{s}}\right.$ being the particle density and $\rho_{\mathrm{g}}$ the gas density).

$u_{\text {th }}$ is approximately given by

$u_{t h}=A \sqrt{\sigma g d}$,

(Bagnold 1941; Kok et al. 2012; Katra et al. 2014), where

$\sigma=\frac{\rho_{\mathrm{s}}-\rho_{\mathrm{g}}}{\rho_{\mathrm{g}}}$

and $A$ is the dimensionless threshold friction velocity.

Claudin \& Andreotti (2006) proposed a scaling law between the ripple wavelength and the drag length, $L_{\mathrm{drag}}$

$L_{\mathrm{drag}}=d \frac{\rho_{\mathrm{s}}}{\rho_{\mathrm{g}}}$,

and showed it to be a good fit over five orders of magnitude on objects ranging from Mars to Venus to subaqueous ripples on Earth. Use of this scaling law with typical surface gas densities on the comet would result in predicted ripple wavelengths much larger than the size of the nucleus itself. However, the equation for $u_{\mathrm{th}}$ is invalid for grains smaller than $100 \mu \mathrm{m}$, and particularly so on comets, because there is a rapid increase of threshold friction velocity with decreasing particle size caused by interparticle cohesion (Shao \& Lu 2000; Iversen et al. 1976).

A simple comparison of the van der Waals force, for instance, using the equation

$F_{\mathrm{vdW}}=\frac{H d}{12 z_{0}^{2}}$

where $H$ is the Hamaker constant, typically of about $3 \times 10^{-20} \mathrm{~J}$, and $z_{0}$ the particle-to-surface distance, usually assumed to be $0.4 \mathrm{~nm}$ (Zoeteweij et al. (2009), with the gravitational force on the particle is sufficient to illustrate this. It is also instructive to compare this to the drag force acting on a particle at rest, but submerged in a fluid moving with a velocity, $v_{\mathrm{R}}$, that is, Eq. (1). 
Table 1. Comparison of forces and velocities on grains of $1 \mathrm{~mm}$ diameter with a density of $1000 \mathrm{~kg} / \mathrm{m}^{3}$.

\begin{tabular}{llll}
\hline \hline Quantity & Value & Equation source & Notes \\
\hline$u^{*}{ }_{\mathrm{t}}$ & $6.88 \mathrm{~m} / \mathrm{s}$ & Katra et al. & Const. $=0.1$. No cohesive forces. $g=1.15 \times 10^{-4} \mathrm{~m} / \mathrm{s}^{2}$ \\
$u^{*}{ }_{\mathrm{t}}$ & $335.64 \mathrm{~m} / \mathrm{s}$ & Shao and Lu & $\gamma=3 \times 10^{-4}$ and $p=30$ nanobar. \\
$F_{\mathrm{g}}$ & $8.12 \times 10^{-11} \mathrm{~N}$ & & Simple gravity calculation. \\
$F_{\mathrm{ddW}}$ & $1.56 \times 10^{-5} \mathrm{~N}$ & Zoeteweij et al. & \\
$F_{\mathrm{c}}$ & $1.8 \times 10^{-7} \mathrm{~N}$ & Scheeres et al. & $S=0.1$ \\
$F_{\mathrm{sl}}$ & $5.8 \times 10^{-9} \mathrm{~N}$ & Shao and Lu & Drag force corresponding to Shao and $\mathrm{Lu} ; C_{\mathrm{D}}=4$. \\
\hline
\end{tabular}

This shows that $F_{\mathrm{vdW}}$ is several orders of magnitude larger than the gravitational force and also much larger than $F_{\mathrm{D}}$ for realistic values of the cometary gas density and velocity even when neglecting the reduction in local gas velocity caused by friction with the surface.

The equation for $F_{\mathrm{vdW}}$ above applies to a dust particle on a flat smooth surface which, however, is not applicable for particulate surfaces on comets. The key question, though, is how much this force is reduced by the specific conditions. There are several questions that are poorly understood at this point. For example:

1. The cross-sectional area of the contact points between surface particles is unknown.

2. The influence of torque on the probably highly fragile particles is unknown.

3. Saffman lift force is caused by the sharp gradient in the fluid velocity above a particle bed, which creates a lower pressure above the particle than below it as a consequence of the Bernoulli effect (Kok et al. 2012). This can lower the effective cohesive force.

4. The effects of local turbulence may be strong, especially in the irregular structure of the neck.

A simple expression to fit the experimental data presented in Greeley \& Iversen (1985) was produced by Shao \& Lu (2000):

$u_{\mathrm{th}}=\sqrt{A_{\mathrm{N}}\left(\sigma g d+\frac{\gamma}{\rho d}\right)}$,

where the second term is intended to account for the cohesive forces. This equation was used by Thomas et al. (2015) to estimate the gas velocities needed to produce saltons on 67P. When applied to a low-gravity regime such as the cometary nucleus, the cohesive term becomes strongly dominant even for $100 \mu \mathrm{m}$ particles (as argued by Cheng et al. 2013). In Fig. 19 we show this graphically for low pressures over the particle size range $1-10000 \mu \mathrm{m}$

Scheeres et al. (2010) suggested use of the equation

$F_{\mathrm{c}}=1.8 \times 10^{-2} S^{2} d$,

where $S$ is a numerical constant approximately equal to 0.1 , to compute the cohesive forces in lunar regolith and argued that this will underestimate the van der Waals force for particles on asteroids or in micro-gravity. (We note that Scheeres et al. 2010 used a value for the Hamaker constant roughly $50 \%$ greater than given above in deriving the numerical constant in Eq. (17).)

A comparison of the forces and fluid threshold velocity values computed from the different equations is shown in Table 1. The table illustrates the difference in magnitude between gravitational and cohesive forces on comets. It also shows that two approaches to estimating the cohesive forces for regoliths produce significantly different results. The Shao \& Lu (2000) formulation leads to forces lower by factors of 30 than the

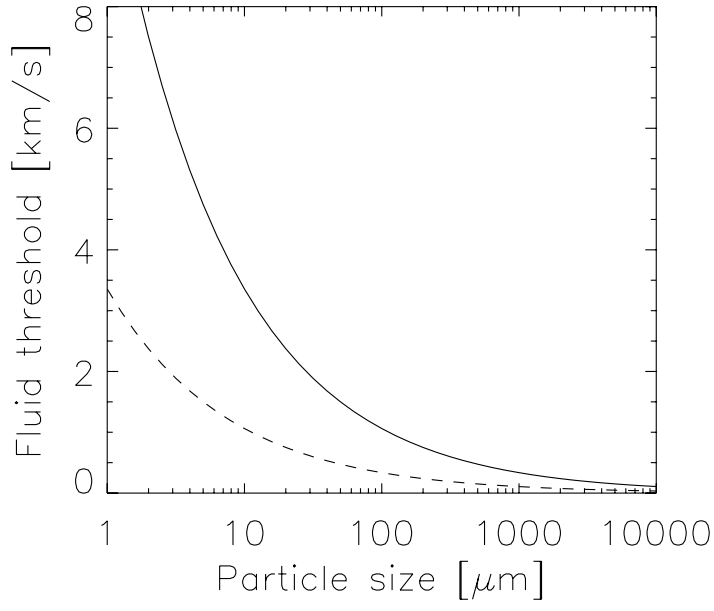

Fig. 19. Fluid threshold velocity calculated using the Shao-Lu formulation with values for gravity appropriate for $67 \mathrm{P}\left(g=1.55 \times 10^{-4} \mathrm{~m} \mathrm{~s}^{-2}\right)$. The velocity rises rapidly as the particle size decreases because of cohesive forces. Gas pressures of $0.003 \mathrm{~Pa}$ (solid line) and $0.03 \mathrm{~Pa}$ (dashed line) are shown.

Scheeres et al. (2010) formulation, which, in turn, leads to a similar reduction in the gas pressure needed to mobilize the grains. The concept of cometary "saltation" in its simplest form probably needs cohesive forces to be closer to the Shao \& $\mathrm{Lu}$ (2000) description to be feasible.

The gas pressures and velocities needed for the Shao \& $\mathrm{Lu}$ (2000) formulation to be sufficient are still fairly extreme. To match the drag force, a pressure of 30 nanobar is needed with gas velocities exceeding $300 \mathrm{~m} / \mathrm{s}$. It should also be clear that to produce aeolian effects on the nucleus, a gas flow is required with a significant component parallel to the local surface. If the comet were to be a uniformly emitting sphere, then any non-radial flow would be limited to molecules emitted non-radially inside a lowdensity layer (i.e., low production rates would be needed in a non-collisional regime). The introduction of insolation-driven sublimation that is homogeneous over the nucleus produces a lateral component. As has been shown by several previous authors (e.g., Kitamura 1987), this lateral motion is strongly enhanced if outgassing is inhomogeneous (i.e., for jet-like structures). In the case of 67P, the geometry of the nucleus, and particularly the neck, can produce additional effects as a result of both the insolation distribution and the partial confinement of the expanding gas. The "walls" of the neck (Hathor and Seth) are also potential gas sources.

From a modeling perspective, the problem can become extremely complex rather quickly as the level of complexity of the source is increased. A detailed study of gas flow for all ventifacts is beyond the scope of this paper because it would require a full assessment of gas and dust sources from multiple datasets and, consequently, a large number of simulations. Our aim here 


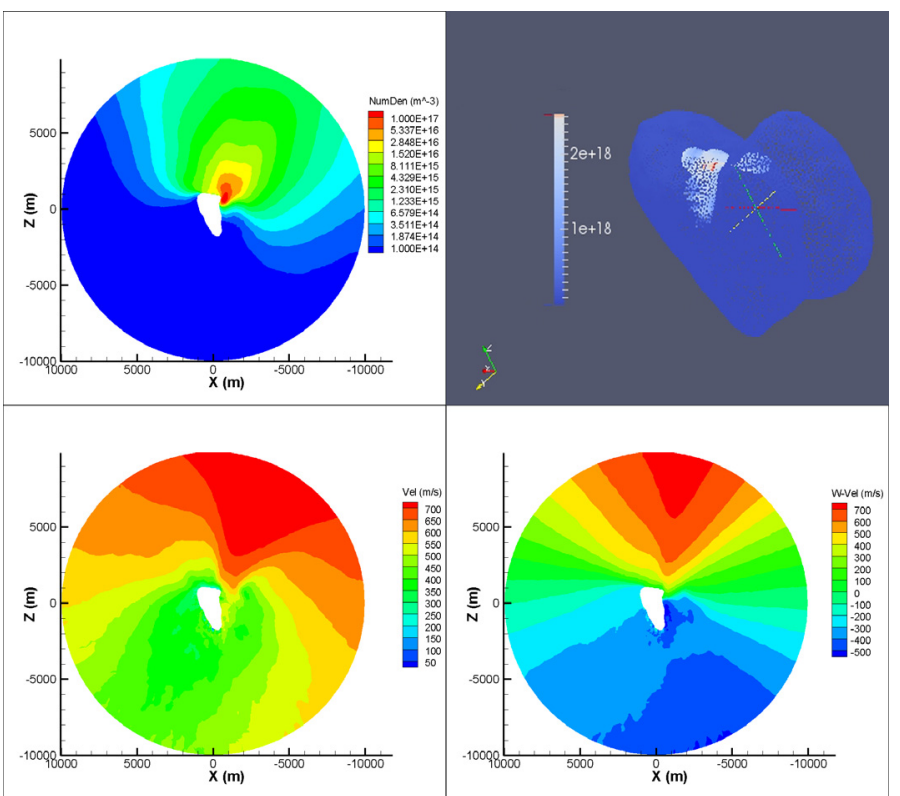

Fig. 20. Gas dynamics model and results. Upper right: the surface density boundary condition used for the calculation. Upper left: the resulting density in the $x-z$ plane at a y position of $+900 \mathrm{~m}$ providing a slice directly above the putative aeolian ripples. The white area indicates that the slice cuts through the nucleus to the left of the ripples. Lower left: the gas speed in the domain. Note the high speed just to the right of the nucleus at the approximate position of the ripples. Lower right: the $y$ velocity of the gas speed. This illustrates that the gas is mostly directed downward, in good agreement with the ripple orientation at velocities of around $500 \mathrm{~m} / \mathrm{s}$. is to establish some working values for gas density and velocity within the neck region and to compare these with the values needed to initiate particle motion. Hence, our model set-up is relatively simple.

To investigate the gas velocities and densities that might be expected in the neck region of the nucleus, we ran a simulation using the 3D DSMC code described in Sect. 3.2. However, here we adopted a highly inhomogeneous boundary condition. To determine typical expected velocities, we set up a calculation in which the neck of the nucleus was strongly active, but the dune field and other areas of the nucleus were only weakly active. Equation (9) was used to set the boundary condition, with areas in the neck set to a flux equal to a $10 \%$ active fraction with other areas set to $0.3 \%$. The surface density arising from the boundary conditions is shown in Fig. 20 (upper right panel). The variation across the surface shown in Fig. 20 arises from the variation in the angle of incidence. The resulting density in the $x-z$ plane at a $y$ position of $+900 \mathrm{~m}$ is shown in the upper left panel. The $y=900 \mathrm{~m}$ position provides a slice through the domain directly above the putative aeolian ripples. The white area indicates that the slice only cuts through the nucleus to the left of the ripples. This orientation is different from that of the upper right panel. The orientation in the upper right panel was chosen to give a better view of the boundary condition. The axes in the upper right panel, however, show the orientation, and the white area in the other panels clearly corresponds to the body of the nucleus. The slice does not cut through the head. The gas density shows a substantial density gradient in the vicinity of the ripples, with the density dropping two orders of magnitude over a distance of approximately $1 \mathrm{~km}$. To the lower left, the gas speed in the domain is shown. The expansion and acceleration of the gas from the neck into the coma is clear, as is the lateral expansion. The gas speed just to the right of the nucleus at the approximate position of the ripples is high. This, however, is the magnitude of the velocity, and therefore we show in the lower right panel the y component of the gas velocity. This illustrates that the gas is mostly directed downward and hence agrees well with the ripple orientation. To some extent, the irregular shape of the nucleus, combined with the lateral expansion toward the nightside, partially funnels the gas over the ripple area. We conclude from this that outgassing from the neck can produce highspeed $(500 \mathrm{~m} / \mathrm{s})$ near-surface gas flow orthogonal to the ripple orientation. Quantitatively, the results indicate the need for better understanding of the cohesive forces and detailed knowledge of the gas source. Assuming a gas density of $5 \times 10^{16} \mathrm{~m}^{-3}$ (close to the maximum shown in the upper left panel of Fig. 20), then the shear velocity at a fluid threshold for $d=1 \mathrm{~cm}$ particles using the Shao and Lu formulation is $528 \mathrm{~m} / \mathrm{s}$, which is comparable to the computed wind speed and indicates that particles of this size and larger (cf. Fig. 19) can be lifted. However, as pointed out above, the gas density drops rapidly in the $-z$ direction. A lower local density would lead to an increase in the fluid threshold velocity. This can be compensated for by adapting the positions of the gas sources by bringing them closer to the ripple structure. The closest source in the current simulation is centered approximately $170 \mathrm{~m}$ from the ripples, which clearly indicates that for this mechanism to be effective, the gas sources must be close to the observed features.

Although the calculation indicates that particles can be moved, another question is whether the saltation mechanism can generate ripples. Particles lifted by the gas drag do not necessarily saltate in the manner seen on Mars because of the nearabsence of a gravitational force that would bring the lifted particle back to the surface. Clearly, the lifting mechanism must result in very low ejection velocities to allow the particles to fall back within a few meters on quasi-ballistic trajectories. This is contradictory because the gas drag lifting the particle overcomes cohesive forces that are much stronger than the acting gravitational forces. Hence, one would expect that once the particle is lifted, it is removed from the area by the gas because the main opposing force is now completely absent. It is also important to point out here that we used equations for saltation that are far outside the usual parameter range. Hence, there may be effects that act to reduce the fluid threshold velocity (e.g., Saffman force).

\subsection{Airfall "splash" mechanism}

The previous section has shown that we have extreme requirements to generate sufficient saltons and that, conversely, these saltons must de-couple from the gas flow quickly to re-impact the surface. However, there is an alternative that uses our previous conclusion concerning the importance of airfall. The initial saltons that produce the first "splash" might be airfall particles.

Particles are already being lifted by the sublimation itself, and we have shown that a fraction of these particles fails to escape and will re-impact the nucleus. Although traveling $<1 \mathrm{~m} / \mathrm{s}$, the splash produced by these particles can initiate creep and reptation. There are several attractive elements in this model. First, gas drag to initiate motion, far from an active source, is unnecessary. Second, when particles overcome the very strong van der Waals forces and are lifted from the surface, they enter an extremely high-velocity relatively dense flow in the wind-driven model, where they can be easily swept away. The drag force is weaker than the van der Waals force, and hence any gas flow that overcomes van der Waals forces is much higher than the 
force needed to accelerate the particles to escape velocity. In the airfall-initiation concept, particles are levitated naturally, and gas drag on the particles can be far lower. This implies that the "effective" fluid threshold is not governed by the cohesive term, but mostly by the gravitational term. Hence, much lower gas velocities or much lower densities are required to start the process. Third, the model naturally emplaces larger particles (i.e., those less influenced by van der Waals forces) in the ripple material as a direct result of the airfall mechanism. Greeley et al. (2002) pointed out that grains that comprise ripples are typically coarse $(>500 \mu \mathrm{m})$ and that the coarsest grains are found on the ripple crests. Jerolmack et al. (2006) noted that aeolian ripples should be separated into splash ripples, where there is little difference between particles on the crests and in the troughs, and coarsegrained ripples, where larger particles are found preferentially on the crests.

We computed the density required at $500 \mathrm{~m} / \mathrm{s}$ flow speed (as found in the gas dynamics calculation) to initiate saltation motion in the absence of cohesion with particles $1 \mathrm{~cm}$ in diameter using Eq. (12). This results in a gas density of $5.6 \times 10^{15}$ molecule $\mathrm{m}^{-3}$ which, when compared with Fig. 20, is a far less extreme requirement.

We are left, however, with trying to understand why such a pristine set of ripples is found only once on 67P and only in the observed place on the nucleus. Clearly, the surface itself cannot be active, as this provides a natural way to destroy the observed pattern. It is at the edge of a region of activity, however, which is probably necessary to provide a gas source. Another aspect is that the surface is also on a gravitational slope. The precise value of this slope cannot yet be completely determined without knowledge of the shape of the southern hemisphere of $67 \mathrm{P}$ (even if an assumption of a uniform internal density distribution is valid). However, based on the SHAP4S shape model, the surface is sloped by an angle, $\alpha$, of $10.2^{\circ}\left( \pm 2.7^{\circ}\right)$ with respect to the local gravitational isopotential (and around $1.5^{\circ}$ lower if centrifugal acceleration is taken into account). In combination with a weak drag force as gas expands laterally around the neck, a concept similar to that shown in Fig. 21 can be envisaged.

Models of the process are clearly required, but as has been shown previously (Anderson \& Haff 1988), this is not trivial when considering the details. The model results of Anderson and Haff cannot be used directly because the impact angle of the initial saltons (assumed to be $8^{\circ}$ with respect to the bed by Anderson and Haff) is likely to be much higher, which will tend to produce more widely distributed ejecta. However, these calculations show that low-impact velocities produce only few ejected particles, with the ejection speed being around $50 \%$ of the impact speed. If we assume a ballistic trajectory for the particles ejected by a splash, then we can easily determine the distance moved, $s$, as a function of the ejection angle, $\theta$, and speed, $v$, from the equation

$s=\frac{2 v^{2} \cos \theta \sin \theta}{g}$

The contour plot in Fig. 22 indicates that ejection angles and speeds required, such that ejecta stay within the confines of the ripple field (i.e., a flight range of $<120 \mathrm{~m}$ ), are easily obtained if airfall impact speeds are below $0.5 \mathrm{~m} / \mathrm{s}$ and if ejection speeds of splashed ejecta are lower than $50 \%$ of the impact speeds.

\subsection{Potential alternative explanations}

The above calculations suggest that wind-driven production of ventifacts remains plausible even though it requires extreme

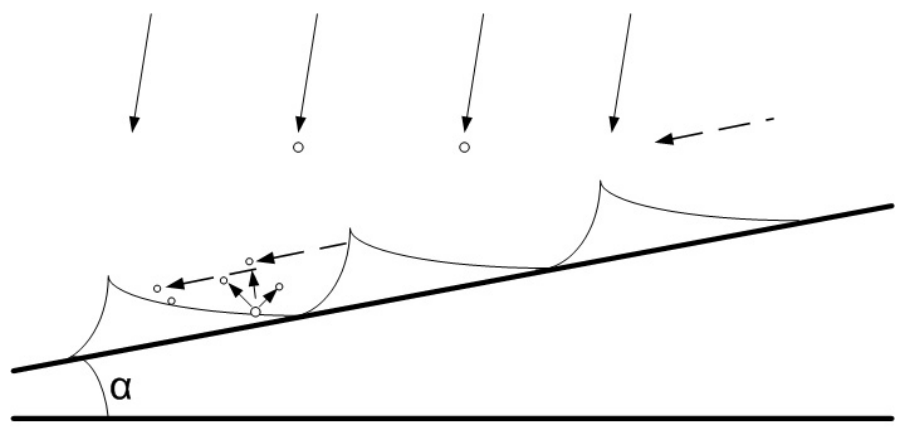

Fig. 21. Schematic drawing of the proposed ripple-field production mechanism. Airfall comes from above, impacting the surface at speeds of around $0.5 \mathrm{~m} / \mathrm{s}$. Slower speed ejecta are produced that creep along the surface, possibly driven by gas drag and/or the gravitational slope.

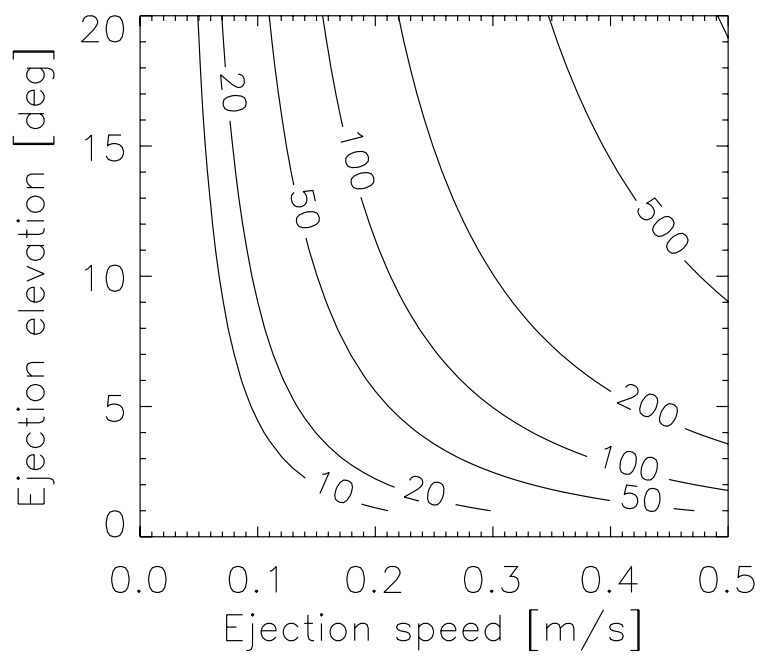

Fig. 22. Distance traveled by a particle ejected from the surface of $67 \mathrm{P}$ on a ballistic trajectory as a function of its initial speed and the ejection angle with respect to the surface. The contours are in $[\mathrm{m}]$. The ripple field on 67P is around $120 \mathrm{~m}$, and hence particles traveling farther than this distance (even in the absence of gas drag) would leave the ripple field.

conditions. We also showed that airfall may be a trigger for surface dust transport, providing a more straightforward and less extreme mechanism. However, there are many uncertainties and difficulties in reaching a final conclusion. Hence, it is probably useful at this point to speculate on alternative non-wind-related mechanisms for the production of the observed features. There are four possibilities:

1. Thin dust-coating over a rough substrate.

2. Gravitational processes.

3. Electrostatic processes.

4. Preferential erosion of the dust surface.

We discuss these in turn below.

It is conceivable that the dune-like structures are a consequence of a conformal coating over a rough substrate. The only way to address this problem is to infer the nature of the substrate from uncoated areas surrounding the dune. In the Maftet region, a fractured but topographically quite smooth terrain is visible near the putative dunes (Fig. 16), which would suggest that the substrate does not produce the observed topography. 
Processes connected to the local gravity, such as local landslides, have two difficulties to overcome. First, these processes do not lead to local topographic maxima, and second, any material motion must overcome the cohesive forces, which, as we have shown above, can be substantially stronger than the gravity force for particles smaller than $1 \mathrm{~cm}$ in diameter. For example, in a gas flow of $500 \mathrm{~m} / \mathrm{s}, 0.5 \mathrm{nbar}$, the Shao and Lu formulation for the cohesive forces and the gravitational force are of similar magnitude for particles $1 \mathrm{~cm}$ in diameter of $1000 \mathrm{~kg} / \mathrm{m}^{3}$ density, with cohesive forces becoming rapidly dominant as the particle size decreases. The formulation of Scheeres et al. for the cohesive forces would give significantly higher cohesive forces.

Electrostatic effects on particles are discussed in the following section in the context of ponded deposits, but there seems to be no obvious way in which such effects can produce dunelike topographic structures, particularly considering the apparent large size of the particles present.

Finally, the smooth material may be active and the dune structure results not from deposition, but from a preferential erosion of the smooth material. As we do not know the initial state, the features we see may simply be a consequence of quasirandom initial condition. Scientifically, this is a highly unsatisfactory ad hoc explanation, but it is difficult to eliminate.

Hence, none of the alternatives offers a particularly attractive explanation. However, explanations for the production of these structures would benefit enormously from any future evidence of changes.

\section{Ponded deposits}

As noted in Thomas et al. (2015), there are several ponded deposits in the Khepry and Aker regions. Morphologically, they follow the description of those seen on 433 Eros (Robinson et al. 2001), being flat-floored and sharply embaying the bounding depression in which they sit (Dombard et al. 2010), although Roberts et al. (2014) stated that fewer than half the pond candidates on 433 Eros have clearly flat floors. The features on 67P are up to $160 \mathrm{~m}$ diameter (see Fig. 23) and therefore similar in size to those seen on Eros (Roberts et al. 2014; their Table 1). The SPC shape model was used to estimate a maximum depth of $35 \mathrm{~m}$ from the depression rim to the floor. On Eros, the ponded terrain is relatively blue. We studied this on 67P and found no significant color difference between the ponded deposits and the surroundings using the five-color data set from which Fig. 23 was taken. At the time of writing, with the southern hemisphere not yet fully illuminated or mapped, these features are only found in the consolidated cometary material of Khepry and Aker.

Four mechanisms for ponded deposit production have been proposed and investigated. Cheng et al. (2002b) proposed that the pond deposit is the result of seismic shaking from impacts. Dombard et al. (2010) have suggested that the ponds form as a consequence of thermal disaggregation of boulder material within the depression in a type of insolation weathering driven by the repeated day-to-night cycling - this mechanism was proposed as a cause of fracturing on 67P by Thomas et al. (2015). The flattening is produced by seismic shaking of ponds in response to impact. Roberts et al. (2014) have criticized this by showing that the pond material follows the underlying topography, which is inconsistent with the material originating by erosion of central boulders. Electrostatic levitation of dust and transport has been proposed and investigated by several authors. Poppe et al. (2012) have pointed out that there is now significant evidence for electrostatically induced dust grain transport above the lunar surface, and they extended previous modeling work to

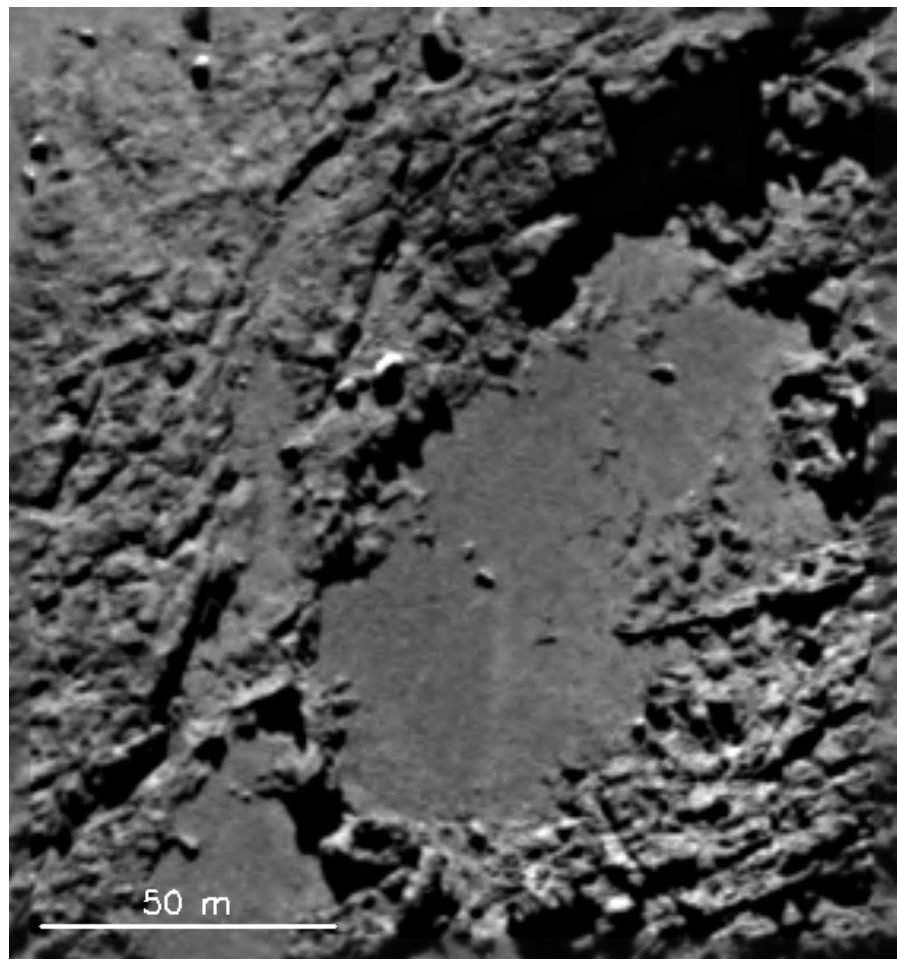

Fig. 23. Ponded deposits in Khepry. Image: NAC_2014-0918T08.07.20.370Z_ID10_1397549000_F22.

include the ponded deposits of Eros and the trapping efficiency of dust grains by craters. They showed that grains will tend to accumulate within crater boundaries as a consequence of the presence of complex fields at crater rims, with larger grains being trapped more efficiently. The main problem, however, is the absence of a well-defined launch mechanism. Micrometeoroid impact has been proposed, but found to be insufficient in the case of Eros (Colwell et al. 2005). For electrostatic lofting, cohesive forces need to be account for, which leads to preferential lifting of intermediate-sized $(15 \mu \mathrm{m})$ grains (Hartzell et al. 2013). This problem may not exist for 67P because grains are being levitated by the sublimation process. Hence, only the preferential transport of these grains into depressions is needed. Poppe et al. (2012) appear to demonstrate that this is feasible, although we note the relatively small scale of the modeled crater $(7 \mathrm{~m}$ diameter) compared to our observed deposits. Finally, Sears et al. (2015) have recently suggested that fluidization associated with degassing should also be considered as a possible explanation, which might in turn be related to similar mechanisms proposed for the production of other features on comets (Belton \& Melosh 2009).

\section{Conclusions and discussion}

There are many lines of evidence suggesting emission of nonescaping $\mathrm{cm}$-sized particles from active areas on the nucleus of 67P. Numerical models show that emission of slow-moving large particles from the Hapi region (the region observed to be active in the early pre-perihelion phase) leads to deposition over much of the northern hemisphere of the nucleus. If large particles rapidly decouple from the gas after ejection from the surface, then particles ejected at speeds of $<0.5 \mathrm{~m} / \mathrm{s}$ fail to escape from the neck and either return to the surface of Hapi or are deposited on the surfaces of Seth and Hathor. On the other hand, particles faster than about $1.0 \mathrm{~m} / \mathrm{s}$ either escape or collide with 
the neck on their way. We therefore have a type of velocity filter in action where only particles in the $0.5-1.0 \mathrm{~m} / \mathrm{s}$ range coat surfaces outside the neck. Particles in the lower half of this range re-impact the northern hemisphere, while particles in the upper half of the range are in a regime where escape and distribution over the entire nucleus occur. The latter cause smaller accumulations of material on the surface.

The observations strongly suggest that airfall is concentrated on the surfaces facing north. However, this does not imply that re-impacting ejected large particles are solely in the $0.5-0.75 \mathrm{~m} / \mathrm{s}$ velocity range. It merely reflects the fact that slightly faster particles are more evenly distributed around the nucleus, which results in lower depths of airfall elsewhere. The re-impacting particles have the potential to be gas sources and can lead to gas emission on a global scale, but with a low production rate as the comet approaches the Sun.

Within the neck, there are structures that are reminiscent of aeolian (coarse-grained) ripples. Estimates of the amplitudeto-wavelength relation from a local shape model $(0.02-0.04)$ are similar to typical values seen on Earth for these structures. However, unlike aeolian ripples seen elsewhere in the solar system, the dominant forces opposing particle motion are cohesive and not gravitational. This makes the entire concept of a "cometary saltation" debatable. On the other hand, the gas flux can be high enough to exceed cohesive forces if the gas sources are close enough. We have shown through gas dynamics modeling that lateral expansion of the sublimed gas can quickly reach $500 \mathrm{~m} / \mathrm{s}$, partially compensating for the extremely low gas densities, although nearby sources are needed to generate sufficient force to mobilize larger particles. The concept remains unproven because of uncertainties in the magnitude of cohesive forces and the effective particle size participating in the process. The conditions required are, however, extreme and require rapid decoupling of the dust from the gas after pick-up. Given the high organic content of the particles, wind-driven saltation may also be opposed by particle bonding (in addition to cohesive forces) in forming an organic matrix or layer over the surface as the material is baked by insolation and modified through interaction with energetic particles. Consequently, particle motion must predominantly involve large particles and needs to occur before the development of any organic crust-like structure.

Given the difficulties involved, we proposed an alternative mechanism where reptation or creep is initiated by airfall. Simple calculations indicate that this mechanism is viable and is attractive because it requires far less extreme drag forces. Impacting airfall disrupts the cohesion. Then the combination of a strong (but not extreme) local gas source and a significant local gravitational slope leads to ripple production.

There is evidence for transport of particles elsewhere on the nucleus particularly in the Maftet region on the head. There are dune-like formations up to $2.5 \mathrm{~m}$ high, which show a preferential orientation (La Forgia et al. 2015), and, in some cases, pitted surfaces on slopes facing south. We suggest the following mechanism for their production.

Airfall deposits an insulating layer of $\mathrm{cm}$-sized particles on most of the head of the nucleus. Outcrops of weakly consolidated material remain mostly uncovered because of the geometry of the airfall. These outcrops form slopes that are roughly orthogonal to the sun direction at midday. Hence, they receive maximum insolation and outgas with relatively high production rates pre-perihelion. The gas flow is sufficient to move the airfall deposit locally. If lateral gas flow is responsible, then the gas sources must be extremely close by (we estimate $<20 \mathrm{~m}$ in some cases) to produce a sufficiently large gas flux parallel to the local surface. Additional particles are added to the dune-like formation from the emission of the outcrop. This material might contain icy chunks of volatiles and/or super-volatiles from the outcrop that can form a volatile source for pit production. On the basis of this hypothesis, we predict that position X in Fig. 15 (and similar outcrops in the same region) is or has been a significant recent source of gas.

Many details of the mechanisms involved remain to be worked out, but it is clear that transport and re-distribution of large particles is an important process in defining the surface properties of a significant fraction of the nucleus.

Acknowledgements. OSIRIS was built by a consortium of the Max-PlanckInstitut für Sonnensystemforschung, in Göttingen, Germany, CISAS-University of Padova, Italy, the Laboratoire d'Astrophysique de Marseille, France, the Instituto de Astrofisica de Andalucia, CSIC, Granada, Spain, the Research and Scientific Support Department of the European Space Agency, Noordwijk, The Netherlands, the Instituto Nacional de Tecnica Aeroespacial, Madrid, Spain, the Universidad Politechnica de Madrid, Spain, the Department of Physics and Astronomy of Uppsala University, Sweden, and the Institut für Datentechnik und Kommunikationsnetze der Technischen Universität Braunschweig, Germany. The support of the national funding agencies of Germany (DLR), France (CNES), Italy (ASI), Spain (MEC), Sweden (SNSB), and the ESA Technical Directorate is gratefully acknowledged. The team from the University of Bern is supported through the Swiss National Science Foundation (grant no. 200020_152560) and through NCCR PlanetS.

\section{References}

A'Hearn, M. F., Belton, M. J. S., Delamere, W. A., et al. 2011, Science, 332, 1396

Anderson, R. S., \& Haff, P. K. 1988, Science, 241, 820

Andreotti, B., Claudin, P., \& Pouliquen, O. 2006, Phys. Rev. Lett., 96, 028001

Auger, A.-T., Groussin, O., Jorda, L., et al. 2015, A\&A, submitted DOI: 10.1051/0004-6361/201525947

Bagnold, R. 1941, The Physics of Blown Sand and Desert Dunes

Belton, M. J. S., \& Melosh, J. 2009, Icarus, 200, 280

Bieler, A., Fougere, N., Tóth, G., V., T., \& Combi, M. 2015, A\&A, submitted, 26178

Capaccioni, F., Coradini, A., Filacchione, G., et al. 2015, Science, 347, 628

Carrasco, N., Schmitz-Afonso, I., Bonnet, J.-Y., et al. 2009, J. Phys. Chem. A, 113,11195

Cheng, A. F., Barnouin-Jha, O., Prockter, L., et al. 2002a, Icarus, 155, 51

Cheng, A. F., Izenberg, N., Chapman, C. R., \& Zuber, M. T. 2002b, Meteoritics and Planetary Science, 37, 1095

Cheng, A. F., Lisse, C. M., \& A'Hearn, M. 2013, Icarus, 222, 808

Claudin, P., \& Andreotti, B. 2006, Earth and Planetary Science Letters, 252, 30

Colwell, J. E., Gulbis, A. A. S., Horányi, M., \& Robertson, S. 2005, Icarus, 175 , 159

Crifo, J.-F., Loukianov, G. A., Rodionov, A. V., \& Zakharov, V. V. 2005, Icarus, 176,192

Davidsson, B. J. R., Gutiérrez, P. J., Groussin, O., et al. 2013, Icarus, 224, 154

Dombard, A. J., Barnouin, O. S., Prockter, L. M., \& Thomas, P. C. 2010, Icarus, 210,713

El-Maarry, M., Thomas, N., Giacomini, L., Massironi, M., \& Pajola, M. 2015, A\&A, accepted

Emerich, C., Lamarre, J. M., Moroz, V. I., et al. 1987, A\&A, 187, 839

Finklenburg, S., Thomas, N., Su, C. C., \& Wu, J.-S. 2014, Icarus, 236, 9

Fulle, M. 1997, A\&A, 325, 1237

Gaskell, R. W., Barnouin-Jha, O. S., Scheeres, D. J., et al. 2008, Meteoritics and Planetary Science, 43, 1049

Gombosi, T. I., Cravens, T. E., \& Nagy, A. F. 1985, ApJ, 293, 328

Greeley, R., \& Iversen, J. D. 1985, Wind as a geological process on Earth, Mars, Venus and Titan (Cambridge: Cambridge University Press)

Greeley, R., Bridges, N. T., Kuzmin, R. O., \& Laity, J. E. 2002, J. Geophys. Res (Planets), 107, 5005

Groussin, O., Sunshine, J. M., Feaga, L. M., et al. 2013, Icarus, 222, 580

Gulkis, S., Allen, M., von Allmen, P., et al. 2015, Science, 347, 709

Harmon, J. K., Nolan, M. C., Ostro, S. J., \& Campbell, D. B. 2004, Radar studies of comet nuclei and grain comae, eds. M. C. Festou, H. Keller, \& H. Weaver, 265

Hartzell, C. M., Wang, X., Scheeres, D. J., \& Horányi, M. 2013, Geophys. Res. Lett., 40, 1038

Hermalyn, B., Farnham, T. L., Collins, S. M., et al. 2013, Icarus, 222, 625

Huebner, W. F., Benkhoff, J., Capria, M.-T., et al. 2006, Heat and Gas Diffusion in Comet Nuclei 
8 Keller, H. U., Barbieri, C. Lamy, P., et al. 2007, Space Sci. Rev., 128,

9 Keller, H. U., Mottola, S., Davidsson, B., et al. 2015, A\&A, accepted

10 Kitamura, Y. 1987, Icarus, 72, 555

11 Kok, J. F., Parteli, E. J. R., Michaels, T. I., \& Karam, D. B. 2012, Rep. Prog. Phys., 75, 106901

Kömle, N. I., Kargl, G., Thiel, K., \& Seiferlin, K. 1996, Planet. Space Sci., 44, 675

Kührt, E., \& Keller, H. U. 1994, Icarus, 109, 121

Kührt, E., Knollenberg, J., \& Keller, H. U. 1997, Planet. Space Sci., 45, 665

La Forgia, F., Giacomini, L., Lazzarin, M., et al. 2015, A\&A, accepted

Lien, D. J. 1990, ApJ, 355, 680

Massironi, M., Simioni, E., Marzari, F., Cremonese, G., \& Giacomini, L. 2015, Nature, submitted

Moehlmann, D. 1994, Planet. Space Sci., 42, 933

Mottola, S., Jaumann, R., Schröder, S., et al. 2015, Lunar and Planetary Science Conference, 46, 2308

Pajola, M., Vincent, J.-B., Lee, J.-C., et al. 2015, A\&A, accepted

Poch, O., Pommerol, A., Jost, B., et al. 2015, Icarus, submitted

Pommerol, A., Jost, B., Poch, O., et al. 2015, Plan. Space Sci., in press

Poppe, A. R., Piquette, M., Likhanskii, A., \& Horányi, M. 2012, Icarus, 221, 135

Preusker, F., Scholten, F., Knollenberg, J., et al. 2012, Planet. Space Sci., 66, 54 Prialnik, D., \& Bar-Nun, A. 1990, ApJ, 363, 274

Prigozhin, L. 1999, Phys. Rev. E, 60, 729

Richter, K., \& Keller, H. U. 1995, Icarus, 114, 355

Roberts, J. H., Kahn, E. G., Barnouin, O. S., et al. 2014, Meteoritics and Planetary Science, 49, 1735

Robinson, M. S., Thomas, P. C., Veverka, J., Murchie, S., \& Carcich, B. 2001, Nature, 413, 396

Rotundi, A., Sierks, H., Della Corte, V., et al. 2015, Science, 347, 3905

Scheeres, D. J., Hartzell, C. M., Sánchez, P., \& Swift, M. 2010, Icarus, 210, 968

Sears, D. W. G., Tornabene, L. L., Osinski, G. R., Hughes, S. S., \& Heldmann,

J. L. 2015, Planet. Space Sci., accepted

Shao, Y., \& Lu, H. 2000, J. Geophys. Res., 105, 22437

Sierks, H., Barbieri, C., Lamy, P. L., et al. 2015, Science, 347, 1044

Snodgrass, C., Tubiana, C., Bramich, D. M., et al. 2013, A\&A, 557, A33

Su, C. C. 2013, Ph.D. Thesis, National Chiao Tung Univ., Taiwan

Thomas, N., Sierks, H., Barbieri, C., et al. 2015, Science, 347, 440

Werner, R. A., \& Scheeres, D. J. 1997, Celestial Mechanics and Dynamical Astronomy, 65, 313

Wu, J.-S., \& Lian, Y. 2003, Computers \& Fluids, 32, 1133

Wu, J.-S., \& Tseng, K.-C. 2005, Int. J. Num. Methods Eng., 63, 37

Wu, J.-S., Tseng, K.-C., \& Wu, F.-Y. 2004, Comput. Phys. Comm., 162, 166

Zoeteweij, M., van der Donck, J., \& Versluis, R. 2009, J. Adhesion Science and Technology, 23

1 Physikalisches Institut, Universität Bern, Sidlerstrasse 5, 3012 Bern e-mail: nicolas.thomas@space.unibe.ch
2 Department of Physics and Astronomy, Uppsala University, 75120 Uppsala, Sweden

3 LESIA, Obs. de Paris, CNRS, Univ Paris 06, Univ. Paris-Diderot, 5 place J. Janssen, 92195 Meudon, France

4 Dipartimento di Geoscienze, University of Padova, via G. Gradenigo 6, 35131 Padova, Italy

5 Deutsches Zentrum für Luft- und Raumfahrt (DLR), Institut für Planetenforschung, Asteroiden und Kometen, Rutherfordstrasse 2, 12489 Berlin, Germany

${ }^{6}$ National Central University, Graduate Institute of Astronomy, 300 Chung-Da Rd, 32054 Chung-Li, Taiwan

${ }^{7}$ Laboratoire d'Astrophysique de Marseille , 38 Rue de Frederic Joliot-Curie, 13388 Marseille Cedex 13, France

8 Institute for Geophysics and Extraterrestrial Physics, TU Braunschweig, 38106 Braunschweig, Germany

9 Centro di Ateneo di Studi ed Attivitia Spaziali, Giuseppe Colombo (CISAS), University of Padova, via Venezia 15, 35131 Padova, Italy

10 Department of Mechanical Engineering, National Chiao Tung University, 1001 Ta-Hsueh Road, 30010 Hsinchu, Taiwan

11 Max-Planck-Institut für Sonnensystemforschung, Justus-vonLiebig-Weg, 3, 37077 Göttingen, Germany

12 Dipartimento di Fisica e Astronomia G. Galilei, Vicolo dellÓsservatorio 5, 35122 Padova, Italy

13 International Space Science Institute, Hallerstrasse 6, 3012 Bern, Switzerland and Centro de Astrobiologíca, CSIC-INTA, 28850 Torrejon de Ardoz, Madrid, Spain

${ }^{14}$ Scientific Support Office, European Space Agency, 2201 Noordwijk, The Netherlands

15 Department of Physics and Astronomy, Uppsala University, Box 516, 75120 Uppsala, Sweden and PAS Space Research Center, Bartycka 18A, 00716 Warszawa, Poland

16 Department of Astronomy, University of Maryland, College Park, MD, 20742-2421, USA

17 LATMOS, CNRS/UVSQ/IPSL, 11 Boulevard d'Alembert, 78280 Guyancourt, France

18 INAF-Osservatorio Astronomico di Padova, Vicolo dell Osservatorio 5, 35122 Padova, Italy

19 CNR-IFN UOS Padova LUXOR, via Trasea 7, 35131 Padova, Italy

20 Department of Mechanical Engineering University of Padova, via Venezia 1, 35131 Padova, Italy

21 UNITN, Universitá di Trento, via Mesiano 77, 38100 Trento, Italy

22 INAF-Osservatorio Astronomico, via Tiepolo 11, 34014 Trieste, Italy

23 Instituto de Astrofisica de Andalucia (CSIC), c/ Glorieta de la Astronomia s/n, 18008 Granada, Spain

${ }^{24}$ Science Operations Department, European Space Astronomy Centre/ESA, PO Box 78, 28691 Villanueva de la Canada, Madrid, Spain

25 Institut für Datentechnik und Kommunikationsnetze, 38106 Braunschweig, Germany 